\title{
高效手性膦配体的研究进展
}

\author{
赵文献*,a,b杨代月 $b$ 张玉华 ${ }^{c}$ \\ ( ${ }^{a}$ 商丘师范学院化学化工学院 商丘 476000) \\ ( $b$ 郑州大学化学与分子工程学院 郑州 450000) \\ ( ${ }^{c}$ 商丘市环境监测站 商丘 476000)
}

\begin{abstract}
摘要 手性膦配体的合成及应用是不对称有机反应中的重要研究领域. 按照手性膦配体的手性中心以及配体结构的不 同，对近二十年来在不对称合成中所报道的高效手性膦配体的研究进展进行了综述，并对该类配体的发展前景进行了 展望.

关键词 手性; 膦配体; 不对称合成
\end{abstract}

\section{Progress in Efficient Chiral Phosphine Ligand}

\author{
Zhao, Wenxian $^{*, a, b} \quad$ Yang, Daiyue $^{b} \quad$ Zhang, Yuhua $^{c}$ \\ ( ${ }^{a}$ College of Chemistry and Chemical Engineering, Shangqiu Normal University, Shangqiu 476000) \\ ( ${ }^{b}$ College of Chemistry and Molecular Engineering, Zhengzhou University, Zhengzhou 450000) \\ ( ${ }^{c}$ Shangqiu Environmental Monitoring Station, Shangqiu 476000)
}

\begin{abstract}
Synthesis and application of chiral phosphine ligand is an important research area in the field of asymmetric synthesis. According to the chiral center and the structural characteristics, the recent development of efficient chiral phosphine ligand in asymmetric synthesis is reviewed, and the prospects of chiral phosphine ligand are also discussed.
\end{abstract}

Keywords chiral; phosphine ligand; asymmetric synthesis

历史上第一个手性膦配体是由 Knowles ${ }^{[1]}$ 及 Horne 等 ${ }^{[2]}$ 报道合成的. 迄今为止, 已相继合成出数千个手性 膦配体, 但只有少数手性膦配体得到广泛应用, 实现了 在香料、药物、食品添加剂等方面的工业化生 产 $^{[3 \sim 7]}$. 手性膦配体在不对称合成中起着重要的作用. $\mathrm{Kagan}^{[8]}$ 认为一个好的手性膦配体应该具有以下条件: (1)原料廉价易得; (2)具有较大的刚性骨架; (3)合成步骤 简单; (4)合成过程中不经过拆分; (5)可以同时得到一对 对映体. 本文对近二十年来在不对称合成中报道的手性 膦配体进行了归纳、总结, 并就其自身的优缺点和今后 的发展趋势进行了全面的讨论.

\section{1 膦配体}

一般认为, 手性催化剂的不对称单元应尽可能的靠 近化合物中心的金属原子 ${ }^{[1]}$, 因此早期的膦配体的手性
中心大多数位于磷原子上. 手性膦配体包括手性单膦配 体、手性双膦配体、碳中心膦配体等几种类型.

\section{1 单膦配体}

手性单膦配体主要是手性碳骨架的单磷配体. 早在 1968 年 Knowles ${ }^{[1]}$ 和 Horner 等 ${ }^{[2]}$ 将手性单齿磷配体应用 到不对称催化反应，但是单齿磷配体很快被双齿磷配体 替代. 直到 1991 年 Hayashi 等 ${ }^{[9]}$ 成功地将单齿 MOP 配 体应用于 Pd 催化的烯丙基取代反应，人们又重新关注 单齿膦配体的研究.

单膦配体根据磷原子的成键不同分为膦配体、亚膦 酸酷配体、亚磷酸酷配体、亚磷酰胺酷配体以及其他类 型的单膦配体(图 1).

最早人们对膦配体的合成限于用各种取代基取代 Wilkinson 催化剂的苯基, 合成膦手性膦化合物并用于

* E-mail: zhwx2595126@163.com

Received March 4, 2016; revised May 30, 2016; published online June 20, 2016.

Project supported by the National Natural Science Foundation of China (Nos. 20972091, 21172139), and the Key Science Research of Education Committee in Henan Province (No.16A150020).

国家自然科学基金(Nos. 20972091, 21172139)、河南省高等学校重点科研(No. 16A150020)资助项目. 


\begin{tabular}{|c|c|c|}
\hline $\begin{array}{l}R_{\backslash}-P-R \\
R^{\prime}\end{array}$ & $\begin{array}{l}\mathrm{RO}^{-} \mathrm{P}-\mathrm{R} \\
\mathrm{RO}^{-}\end{array}$ & $\begin{array}{l}\mathrm{RO}^{-} \mathrm{P}-\mathrm{OR} \\
\mathrm{RO}^{-}\end{array}$ \\
\hline 膦配体 & 亚膦酸酯配体 & 亚磷酸酯配体 \\
\hline & $\mathrm{RO}{ }_{\mathrm{D}-\mathrm{N}}-\mathrm{R}$ & \\
\hline & 亚磷酰胺酯配仡 & \\
\hline
\end{tabular}

图 1 不同类型的单膦配体

Figure 1 Different types of single phosphorus ligand

手性催化, 最有效的是 Knowles 合成的手性配体 PAMP 和 CAMP, 但也只有 $60 \%$ 的对映选择性. 在最近几年, 研究者运用不同的合成方法合成了各种类型的手性单 磷配体(图 2). 如手性单膦配体 R-MOP-phen (4)在不对 称氢硅烷化反应中 $e e$ 值可达到 $80 \%$ 以上 ${ }^{[10]}$.

2011 年 Bakos 等 ${ }^{[11]}$ 也报道膦配体 6, 并将其用于衣 康酸二甲酯的不对称氢化反应中, $e e$ 值可以达到 $94.1 \%$. 同时, Kündig 课题组 ${ }^{[12]}$ 将手性亚磷酰胺酯配体 14 应用 于催化前手性的双芳香烃类三羰基铬络合物的不对称 反应中, $e e$ 值可以达到 $98 \%$. 在 2000 年张绪穆课题组 ${ }^{[13]}$ 合成报道了配体 16 18, 用于催化 Baylis-Hillman 反应, 得到的产物的 $e e$ 值只有 $19 \%$; 该课题组 ${ }^{[14]}$ 在 2006 年合 成的配体 13 应用在脱氢氨基酸的不对称氢化反应中, 得到的产品 $e e$ 值可大于 $92.6 \%$. 游书力课题组 ${ }^{[15]}$ 在 2007 年合成并报道了手型单齿亚磷酰胺酯配体 $\mathbf{1 4}$, 并将其 应用到烯丙基亚磺酸盐的异构化和吲哚的分子间不对 称烯丙基烷基化反应中, 分别得到 95\%和 92\%的 $e e$ 值. 以后他们又相继发展出配体 $\mathbf{1 9} \sim \mathbf{2 2}^{[16]}$, 并将其应用在 吡啶、喹啉、异喹啉等杂环化合物的分子内不对称烯丙 基烷基化和吲哚衍生物的分子内不对称烯丙基烷基化 反应中, 得到产物的 $e e$ 值达到 $99 \%$ 和 $93 \% .2013$ 年以后 汤文军课题组 ${ }^{[17]}$ 相继合成了配体 $\mathbf{2 3} \sim \mathbf{2 5}$, 将配体 $\mathbf{2 3}$ 和 24 与钉的配合物应用在催化芳香醛和芳基硼酸的加成 反应中, 得到 ee 值大于 $81 \%$ 的产物, 用在通过 Suzuki-Miyaura 交叉偶合合成手性芳基三氟甲磺酸酯的 反应中, 可以得到 $e e$ 值大于 $87 \%$ 的产物 ${ }^{[18]}$; 汤文军课题 组 ${ }^{[19]}$ 在 2016 年又报道了将配体 $\mathbf{2 5}$ 与钯的配合物, 催化 合成二氧六环木脂衍生物, 得到的产物的 $e e$ 值为 $77 \%$.

单磷配体能够以一配位、二配位或者多配位的形式 与中心金属离子配位并催化反应. 当中心金属离子只能 提供一个配位轨道时, 单磷配体就能够以一个配体同中 心金属离子配位, 进行催化不对称反应. 在多配位的金 属配合物催化的反应中, 单齿磷配体同样能够以多个配 体同时与中心金属离子配位, 这是由于配体没有鳌合作 用, 催化剂的柔性较大, 在催化反应时能够适应不同底 物的要求, 获得更好的选择性. 因此, 可以得到更好的
对映选择性 ${ }^{[20]}$.

\section{2 手性磷双膦配体}

大量研究表明, 由单膦配体制成的过渡金属络合物 催化剂其构型易变, 所以其立体选择性一般不高. 因此 继单膦配体之后, 人们开始将视线转向双膦配体. Knowles 课题组将 PAMP 偶联制备了双齿的 DIPAMP, 这个配体在不对称氢化过程中得到了 $95 \%$ ee 值. 在这 个过程中双齿配体与金属离子发生了鳌合作用，不仅增 加了催化剂结构的刚性，而且提高了催化剂的稳定性. 但是以后关于磷做中心的手性双膦配体很少有报道, 直 到 1995 年配体 26(图 3)的合成, 才进一步开始了对双膦 手性配体的研究, 虽然它的手性中心并不在磷原子上.

配体 26 是由 Burk 小组 ${ }^{[21]}$ 合成的, 并将其与 $\mathrm{Ru}(\mathrm{II})$ 进行配合用于 $\beta$-羰基酯的不对称催化氢化中, 因其构型 稳定, 取得了 $>95 \%$ 的 $e e$ 值. 配体 27 是由 Imamoto 课 题组 ${ }^{[22]}$ 于 1998 年设计合成的, 并在 1999 年将 $\mathbf{2 7}-\mathrm{RuBr}_{2}$ 用于活性羰基化合物的催化氢化. 2011 年, Gridnev 小组 和 Imamoto 小组 ${ }^{[23]}$ 共同报道了手性中心在磷原子上的 双膦配体 29a $\sim 29 \mathrm{c}$, 考察了其用在 $\alpha 、 \beta$-脱氢氨基酸以 及 $\alpha$-取代的烯胺的不对称氢化反应，取得了 $99.9 \%$, $99.8 \%, 95.1 \%$ 的对映选择性. 随后 Imamoto 小组 ${ }^{[24]}$ 在此 基础上对手性配体进行了修饰得到配体 30a, 30b, 在脱 氢氨基酸的不对称氢化反应中表现出很好的催化效果. 在此期间张绪穆课题组 ${ }^{[13]}$ 报道了配体 31 和 32, 并将其 应用在催化 Baylis-Hillman 反应和脱氢氨基酸的不对称 氢化反应中, 得到的产物 $e e$ 值为 $20 \%$ 和 $99 \%$. 然后张绪 穆课题组 ${ }^{[25]}$ 又合成报道了配体 33 和 $\mathbf{3 4}$, 将配体 33 分别 用在 $\alpha 、 \beta$-脱氢氨基酸以及 $\alpha$-取代的烯胺的不对称氢化 反应中, 分别得到了 $e e$ 大于 $95 \%$ 和 $96 \%$ 的产物. 将配体 34 用在 $\beta$-氨基酮的加氢反应中, 根据不同的氢压力、不 同的溶剂、不同的反应时间, 可得到不同的 $e e$ 值的产物, 使其产物的最小的 ee 值达到 $91 \%$ 以上. 汤文军课题 组 ${ }^{[26]}$ 在 2009 年合成了配体 35, 并将其应用在芳基烯烃 的不对称加成上, 得到 $e e$ 值大于 $96 \%$ 的产物. 2013 年汤 文军课题组 ${ }^{[27]}$ 又报道了将这个配体用在 $\beta$-芳基胺的加 成反应和芳基硼酸与含氟芳基酮的加成反应中, 分别得 到 $93 \%$ 和 $85 \%$ 的 $e e$ 值. 在此基础上, 该课题组 ${ }^{[28]}$ 又对这 一类配体进行了修饰, 合成了配体 $\mathbf{3 6}$, 将与钓的配合物 用在催化芳基酮的加成反应中，得到大于 $95 \% e e$ 值的 产物。

这类配体的主要特点是每个 $\mathrm{P}$ 上都连有一个体积大 的烃基(比如叔丁基), 这类配体可以与过渡金属形成一 个具有 $C_{2}$ 不对称轴的五元配合物 28. 该配合物可以在 反应过程中提供不对称环境, 从而使得反应具有高度的 立体选择性. 
<smiles>COc1ccccc1PC1CCCCC1</smiles>

CAMP (1)<smiles></smiles>

2<smiles>c1ccc(P(Cc2cccc3ccccc23)Cc2cccc3ccccc23)cc1</smiles>

3<smiles>COc1ccc2ccc3ccc(-c4ccccc4)cc3c2c1-c1c(-c2ccccc2)ccc2ccc3ccccc3c12</smiles>

4

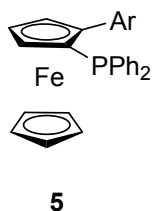

5

手性单齿膦配体<smiles>C[C@@H](C[C@@H](C)Pc1ccccc1)Op1oc2ccc3c(c2c2c4c(ccc2o1)CCCC4)CCCC3</smiles>

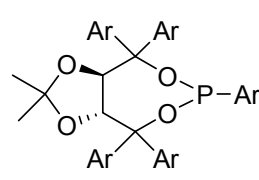

7<smiles>COc1ccccc1PN1[C@@H]2CCCCC2N(C)P1c1ccccc1OC</smiles>

9

手性单齿亚膦酸酯及其类似配体

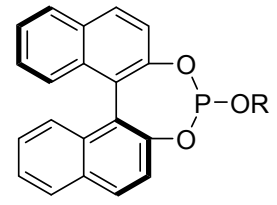

10<smiles>CC1CC[C@@H](C(C)C)C(Op2oc3ccc4ccccc4c3c3c(ccc4ccccc43)o2)C1</smiles>

11<smiles>CC1CC[C@H](C(C)C)[C@H](Op2oc3ccccc3c3ccccc3o2)C1</smiles>

12

手性单齿亚磷酸酯配体<smiles>PN(P)p1oc2ccc3ccccc3c2c2c(ccc3ccccc32)o1</smiles>

13<smiles>CC(c1ccccc1)N(C(C)c1ccccc1)p1oc2ccc3ccccc3c2c2c(ccc3ccccc32)o1</smiles>

14<smiles>[R]N([R])P1OC(c2ccccc2)(c2ccccc2)C(C(C)(C)O)OC1(c1ccccc1)c1ccccc1</smiles>

15

手性单齿亚磷酰胺酯配体<smiles>CC1[C@H](O)[C@@H](O)[C@H](C)P1c1ccccc1</smiles>

16<smiles>CC1[C@H]2OC(C)(C)O[C@@H]2[C@H](C)P1c1ccccc1</smiles>

17

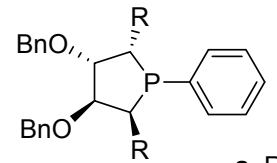

a $\mathrm{R}=\mathrm{CH}_{3}$

18<smiles>COCCCC(C)N([C@H](C)c1ccccc1)P(Oc1ccc2ccccc2c1)Oc1ccc2ccccc2c1-c1ccccc1</smiles>

19<smiles>CC1CCc2ccccc2N1POc1ccc2ccccc2c1-c1c(O)ccc2ccccc12</smiles>

20<smiles>COc1cccc(OC)c1-c1cccc2c1P(C(C)(C)C)[C@@H](C)O2</smiles>

23<smiles>c1ccc(C(c2ccccc2)N(c2ccccc2)p2oc3ccc4ccccc4c3c3c(ccc4ccccc43)o2)cc1</smiles>

21<smiles>C1=Cc2ccccc2N(p2oc3ccc4ccccc4c3c3c(ccc4ccccc43)o2)C1</smiles>

22

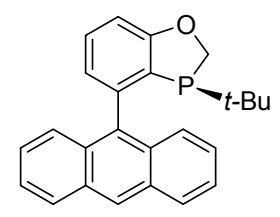

25

图 2 配体 1 25 的结构

Figure 2 Structures of ligands $\mathbf{1} \sim \mathbf{2 5}$ 
<smiles>COc1ccccc1P(CCP(c1ccccc1)c1ccccc1OC)c1ccccc1</smiles>
DIPAMP

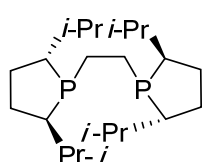

26<smiles>[R]P([2H])CCC[PH]([R])([R])C</smiles>

$27 a \sim 27 e$

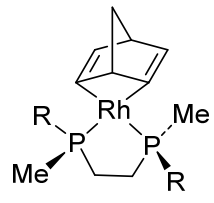

28a $\sim 28 \mathrm{e}$ a: $\mathrm{R}=t-\mathrm{Bu}$

b: $\mathrm{R}=\mathrm{Et}_{3} \mathrm{C}$

c: $R=1$-admantyl d: $\mathrm{R}=\mathrm{C}_{6} \mathrm{H}_{5}$ e: $\mathrm{R}=\mathrm{C}_{6} \mathrm{H}_{11}$

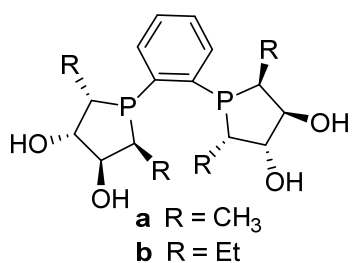

31

a $R=9$-anthryl

b $\mathrm{R}=\mathrm{H}$

c $\mathrm{R}=\mathrm{OMe}$

d $\mathrm{R}=2,6-(\mathrm{MeO})_{2} \mathrm{C}_{6} \mathrm{H}_{3}$
35

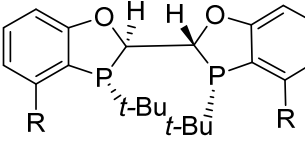<smiles></smiles><smiles>CCCP(C)c1ccccc1[R16](C)(C)C</smiles>

29b

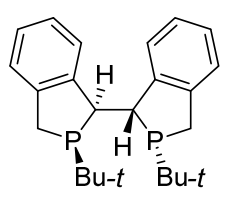

33
34

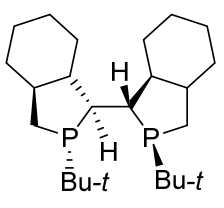

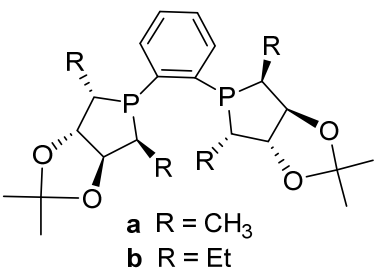

32

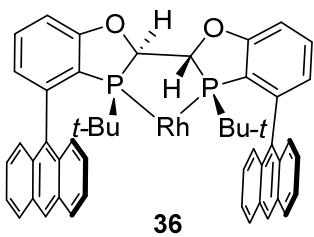

图 3 配体 DIPAMP 及 $26 \sim 36$ 的结构

Figure 3 Structures of ligands DIPAMP and $\mathbf{2 6} \sim \mathbf{3 6}$

\section{C-手性膦配体}

在膦配体发展的基础上, Morrison 认识到: 作为手 性配体, 膦配体碳骨架上的手性也能有效地进行不对称 传递. 这一论点随后被 Kagan 的研究所证实, 他从天然 的酒石酸出发合成得到 $C$-手性的双膦配体 DIOP, 该配 体在不对称催化氢化中取得了比较好的对映选择性. C手性的膦配体比 P-手性的膦配体的制备相对更容易, 结 构的变化性更大.

\section{1 手性碳单膦配体}

1971 年, Morrison 等 ${ }^{[29,30]}$ 第一次合成了手性碳原子 单膦配体 37. 1993 年 Dawson 等 ${ }^{[31]}$ 从手性 $\alpha$-氨基醇合成 了一种新型膦配体 38 , 该配体与过渡金属的络合物在 许多反应中表现出很好的催化性能. 后来 Helmchen $\mathrm{G}^{[32,33]}$ 在此基础上对磷的取代进行了改进, 得到了类似 的配体 39, 常见的此类配体还有配体 $\mathbf{4 0}^{[34]}$. 带有两个 噁唑环的配体 41 也用这样的方法制得 ${ }^{[35]}$, 但是也因为 单膦配体制成的过渡金属络合物手性催化剂构型易变、 立体选择性不高, 单膦配体的发展一直很慢. 直到 $\operatorname{Hamada}^{[36]}$ 与 Chen 等 ${ }^{[37,38]}$ 先后报道了两种相关的手性稠 合双环单膦配体 42 及 $\mathbf{4 3}$, 这样的配体由于具有刚性稠 合双环结构, 构型不易变化. 将其应用在不对称催化反 应中, 均得到大于 $96 \%$ 的 $e e$ 值.

以具有旋光活性的天然产物为原料, 是合成手性膦 的重要途径, 这类工作已经得到相关综述 ${ }^{[39]}$. 近年来 Lugan 等 ${ }^{[40]}$ 以薄荷醇为原料合成了配体 44. 另外手性 $\beta$ -
氨基烷基单膦配体在不对称合成中的应用中也得到了 很大的进展 ${ }^{[41 \sim 43]}$.

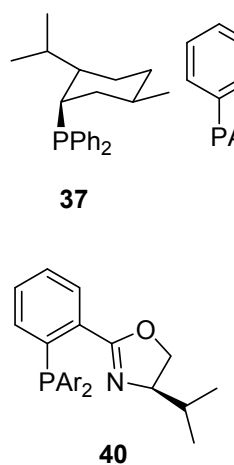

a: $\mathrm{Ar}=\mathrm{Ph}$

b: $\mathrm{Ar}=4-\mathrm{CF}_{3} \mathrm{C}_{6} \mathrm{H}_{4}$ c: $\mathrm{Ar}=3,5-\left(\mathrm{CF}_{3}\right)_{2} \mathrm{C}_{6} \mathrm{H}_{3}$

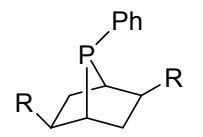

43<smiles></smiles>

39 a: $R=i-\operatorname{Pr}, A r=D B P$ b: $\mathrm{R}=i-\mathrm{Pr}, \mathrm{Ar}=\mathrm{Ph}$ c: $\mathrm{R}=t-\mathrm{Bu}, \mathrm{Ar}=\mathrm{Ph}$<smiles>[R8]=C1C=CC(c2ccccc2C)=CC1</smiles>

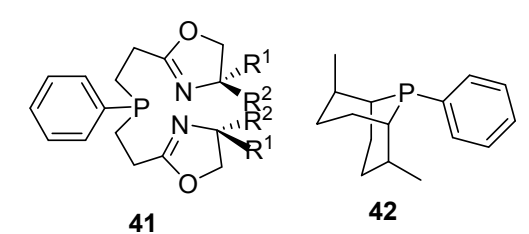
a: $\mathrm{R}^{1}=\mathrm{H}, \mathrm{R}^{2}=\mathrm{C}_{6} \mathrm{H}_{5}$ b: $\mathrm{R}^{1}=\mathrm{CH}\left(\mathrm{CH}_{3}\right)_{2}, \mathrm{R}^{2}=\mathrm{H}$

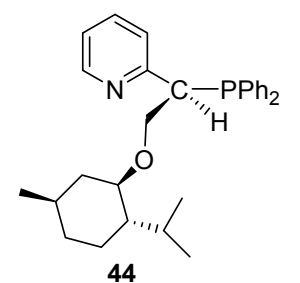

图 4 配体 37 44 的结构

Figure 4 Structures of ligands $37 \sim 44$

\section{2 手性碳双膦配体}

许多低氧化态的金属配合物因为双膦配体的应用 而得以稳定和分离，与单齿膦相比，螯合双膦配体易于 控制配位数和立体构型，增加中心金属原子的碱性，降 低配合物分子间或者分子内的交换过程 ${ }^{[44]}$. 他们在不 
对称合成中具有重大应用，同时也具有巨大的经济效 益.

早在 1972 年, Kagan 课题组 ${ }^{[45,46]}$ 以酒石酸为原料合 成了第一个手性双膦配体 DIOP (45). 配体 45 与过渡金 属铑组成的 $\mathrm{DIOP}-\mathrm{Rh}^{+}$和 $\mathrm{DIOP}-\mathrm{Rh}^{\mathrm{N}}$ 催化剂对各种不饱 和底物的还原都是一种有效的均相催化剂, 且对具有活 性的手性羰基化合物的催化氢化可以在温和的条件下 进行. 实验证明，随着 DIOP 中的 $\mathrm{R}$ 基团体积的增大，催 化剂的催化活性也有很大的增强. 此后一系列 DIOP 的 类似物均被制得 ${ }^{[47,48]}$, 在这些 DIOP 的衍生物中, 以萘 基取代苯基效果较好, 而五元环上的改变对选择性的影 响不大 ${ }^{[49]}$. 如 Achiwa 等 ${ }^{[50]}$ 运用酒石酸衍生物出发合成 了配合物 46 用于烯烃的氢化, 对映选择性高达 $95 \%$. 由 张兆国等 ${ }^{[51]}$ 开发的手性双膦配体 47 , 在催化 $\beta$-酮磷酸 酯的不对称反应中 $e e$ 值高达 $99.9 \%$.

以光学活性的二醇为原料可以合成一系列高选择 性的双膦配体, 如 1993 年 Burk 等 ${ }^{[52 ~ 58]}$ 从二醇出发合成 了 Duphos 型的手性配体 1,2-二膦基苯配体 48 和 1,2-膦 基乙烷配体 49, 将其用于 $\alpha-N$-酰胺基丙烯酸酯催化中 达到 $100 \%$ 的 ee 值. 这两种配体具有下面的特点: 磷原 子上带有富电子的烷基; 依靠 $\mathrm{P}$ 杂环上 2,5 位置的取代 基方向来控制反应物的配位方向, 可以在不对称反应中 获得较高的对映选择性.

根据运用具有光学活性的原料来制备手性配体的 思想, 手性膦的研究取得了很大的进展. 以 $L-4$-差基脯 氨酸合成了 $\mathbf{5 0}^{[59,60]}$, 在用于烯烃的氢化反应中 $e e$ 值可 以达到 $65 \%$; 通过对磷、氮上取代基的改变合成了类似
物 $51 \sim 53^{[61]}$, 在催化酮的氢化还原过程中 $e e$ 值分别达 到了 $66.3 \%, 72.8 \%, 92.0 \%$; 类似的含有氮杂环的配体 AMPP 54 ${ }^{[37]}$ 也已经被合成出来，被用在 $\alpha$-官能团的酮的 氢化还原中, 且取得了 $e e$ 值达到 $90 \%$ 以上的立体选择 性; Rajanbabu 等 ${ }^{[62]}$ 合成了系列配体 $\mathbf{5 5}$, 用于催化氢化 反应中, 得到产物的 $e e$ 值均可以达到 $91 \%$ 以上; 同样由 木糖得到了配体 $\mathbf{5 6}^{[63]}$, 催化反应的 $e e$ 值达到了 $90 \%$ 等.

\section{3 含 $\mathrm{C}_{2}$ 对称轴的膦配体}

在 DIOP 的启发下，人们开始着手合成具有 $C_{2}$ 对称 轴的手性配体. 早在 20 世纪 70 年代 Kagan 和 Dang 就 已经提出, 如果一个具有 $C_{2}$ 对称轴的配体络合到中心 金属离子，则无论反应物从哪一个方向靠近金属离子， 它处在的手性环境都是一样的, 这样就减少了反应的可 能途径 ${ }^{[34]}$. 这样的配体可以和过渡金属形成五元(如 57) ${ }^{[64]}$ 、六元(如 58 $)^{[65]}$ 及七元(如 59 ${ }^{[66 ~ 69]}$ 的螯合环. 在 这些膦配体中, 配体 $48,49,60^{[70 \sim 72]}$ 是较突出的代表, 他们都含有膦五元环，也都具有 $C_{2}$ 手性对称轴，就像配 体 45 和 60 一样, 他们拥有全 $\mathrm{sp}^{2}$ 杂化成键的芳香环骨 架，可以在催化过程中表现出卓越的空间效应，同时还 可以增加催化剂的极性和 Lewis 酸性.

2005 年, 丁奎岭课题组 ${ }^{[73]}$ 报道了配体 61 的合成, 并将其应用在芳基脂的加氢反应中，可以得到 $e e$ 值大 于 $99 \%$ 的产物. Schmid 等 ${ }^{[74]}$ 报道了 62 的合成及其在烯 烃的不对称反应中的应用. 用同样的方法, Rossen 等 $^{[75]}$ 合成了配体 63, 该配体在烯胺的氧化反应中, 其立体选

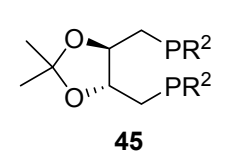

45

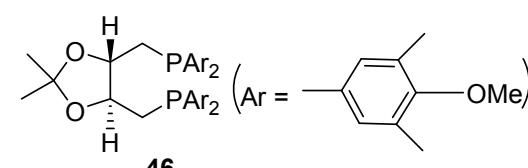

46

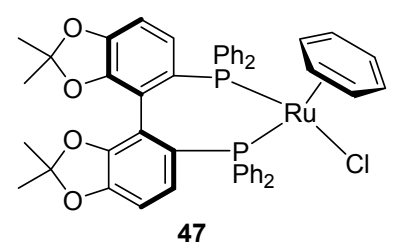

47<smiles>CCCCPC1CC(Cc2ccccc2)N(S(=O)OC)C1</smiles>

51

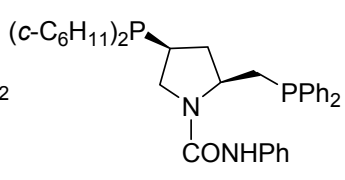

52<smiles>Pc1ccccc1</smiles>

56

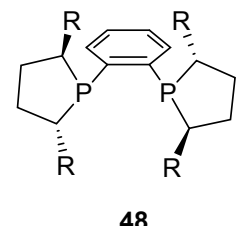

$\left(c-\mathrm{C}_{6} \mathrm{H}_{11}\right)_{2} \mathrm{P} \sum_{\text {COO- } t-\mathrm{Bu}}$

53

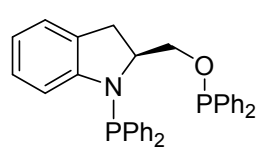

54

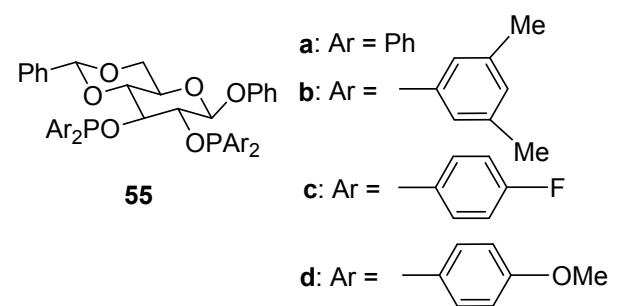

图 5 配体 45 56 的结构

Figure 5 Structures of ligands $\mathbf{4 5} \sim 56$ 


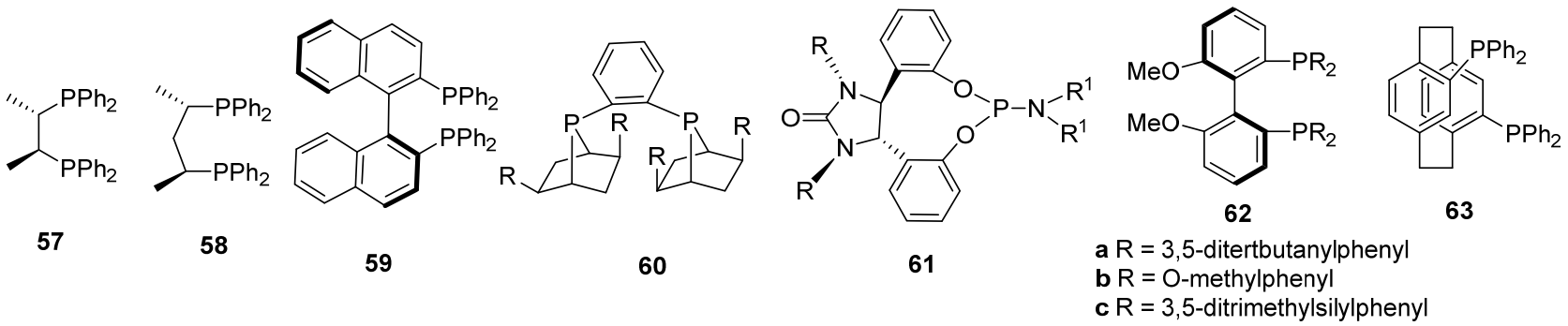

图 6 配体 $57 \sim 63$ 的结构

Figure 6 Structures of ligands $57 \sim 63$

择性高达 $99 \%$ ee. Burk 等 ${ }^{[55,57]}$ 利用配体 48,49 和铑、钉 的螯合物在促进烯胺和酮的不对称催化氢化和立体选 择性方面发挥了优良的催化性能, 对脱氢氨基酸及其衍 生物的不对称氢化，都可以得到很高的 $e e$ 值(接近 $100 \%$ $e e$ ). 在手性配体中, 大多数都是能与中心金属配位成鳌 合物, 尤其是拥有 $C_{2}$ 对称轴的手性双齿膦配体, 它们的 分子有较强的刚性和稳定的构象, 使这一类型的手性配 体所催化的不对称反应其光学活性表现优良.

\section{4 手性中心在两个原子上的膦配体}

尽管人们已经发现了多种优秀的双膦配体, 但是真 正用于工业化的手性膦配体还是很少的, 主要原因在于 手性膦配体合成的难度比较大. 随着手性配体的发展, 人们逐渐发现氮磷、氧磷、双氧膦配体更容易合成且结 构变化更大, 所以这类配体继 $C$-手性膦配体之后成为 了该领域新的研究热点.

\section{1 手性中心在碳、磷原子上的配体}

手性中心同时在碳、磷原子上的配体主要有 64,65 , $\mathbf{6 6}^{[76]}, \mathbf{6 7}^{[77]}$ (图 7). Wills 等在 1994 年报道从光学活性的 胺出发制得配体 64, 65, 66, 并将其用于催化对不饱和 底物的烷基化. 在这些配体中包含了一个重要的结构单 元, 即由一个芳香环并一个 $\mathrm{P}-\mathrm{N}$ 键构成的五元环, 这 保证了在结构中高度的刚性. 在催化反应中发现, 采用 晶体状态的配体的催化效果较非晶体状态的配体的要 好, 如配体 68, 这说明了配体的纯度在反应中起到重要 的作用. 但是由于膦配位上的磷原子对氧敏感, 所以会 采用硼烷对其保护的衍生物.

\section{2 新型螺环骨架膦配体}

根据具有 $C_{2}$ 轴对称性和适当的刚性的双膦配体可 以提高反应的对映面识别能力 ${ }^{[78]}$. Kumar 等 ${ }^{[79]}$ 于 1992 年首次报道了用光学活性的 $c i s$-螺 [4, 4]壬烷-1,6 二醇 69 修饰四氢化铝锂后用于还原芳香酮，得到了 $98 \%$ 的 $e e$ 值. 1996 年陈新滋和蒋耀忠课题组以螺 [4,4]壬烷为母体 合成了 $(1 R, 2 R, 6 R)-1,6$-双 (二苯基膦氧)-螺 [4,4]壬烷[简 称 $(R)$-spirOP, 70] 和 $(1 S, 2 S, 6 S)$-1,6-双 (二苯基膦氧)-螺

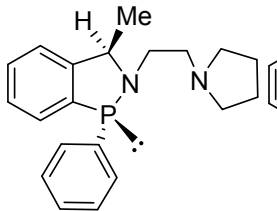

64

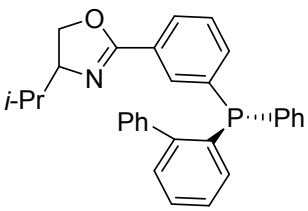

67

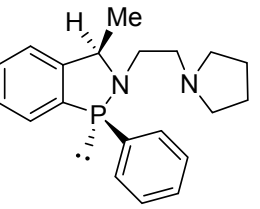

65

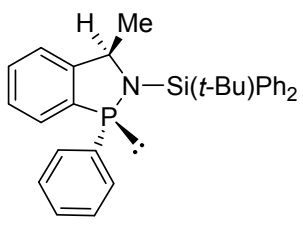

66

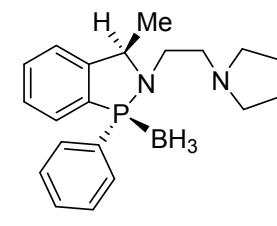

68
图 7 配体 64 68 的结构

Figure 7 Structures of ligands $64 \sim 68$

[4,4]壬烷[简称 $(S)$-spirOP, 71]. 将配体 70 制得相应的铑 催化剂, 在室温、常压下对 2-乙酰氨基丙烯酸进行氢化 可以得到 $96.8 \%$ 的 $e e$ 值, 并且这个配体的高对映选择性 对不同结构的脱氢氨基酸底物具有普遍适应性，如对一 系列(Z)-2-乙酰氨基-3-芳基丙烯酸甲酯的还原，其对映 选择性高达 $90 \% \sim 99.9 \% e e^{[80,81]}$. 丁奎岭课题组 ${ }^{[82]}$ 在此 基础上于 2009 年报道合成了新型螺环骨架的手性膦氮 配体 72, 73, 其在前手性亚胺, 尤其是烷基亚胺的催化 氢化中显示了十分优异的对映选择性 $(e e>91 \%)$. 丁奎 岭课题组 ${ }^{[83]}$ 又在 2012 年合成报道了手性芳香螺缩酮骨 架双膦配体 74 (SKP), 手性 SKP 配体已经在一些金属催 化的不对称反应中取得了成功的应用 ${ }^{[84]}$, 特别是在钯 催化的 Morita-Baylis-Hillman 加和物的不对称烯丙基胺 化反应中，表现出优异的区域和对映选择性(87\% 90\% $e e)$, 以及极高的催化活性 $\left[\mathrm{Pd}_{2}(\mathrm{dba})_{3}\right.$ 用量可降低至万分 之一]. 还将这种方法应用到手性药物依折麦布中间体 的不对称合成中, 为发展绿色环保的药物生产提供了基 础.

\section{5 氮膦、氧膦配体}

这类配体一般是从手性氨基醇和酰胺醇、手性氨基 酸、糖类合成过来的. 手性氨基醇化合物 75 通常以氯作 


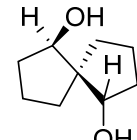

$\mathrm{OH}$

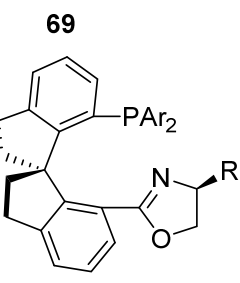

72

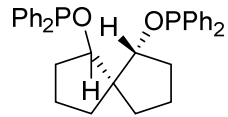

70

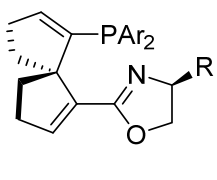

73

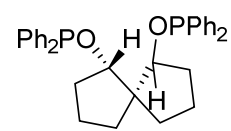

71

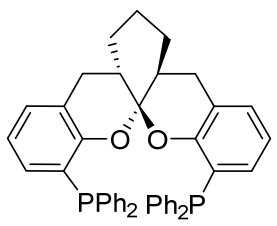

74

图 8 配体 $69 \sim 74$ 的结构

Figure 8 Structures of ligands $69 \sim 74$

辅助配体, 用甲苯作溶剂对 $\mathbf{A}, \mathbf{B}$ 进行了手性催化氢化. 实验表明这类配体可以催化还原 $\mathbf{A}$ 达到 $80 \%$ ～ $89 \%$ ee, 而对 $\mathbf{B}$ 只能达到 $71 \% \sim 77 \%$ ee. 手性酰胺醇是从手性氨 基醇衍生出来的, 含有卤素辅助配体的 76a, 76b 所需的 反应温度低时间短, 对 $\mathbf{A}$ 的催化氢化的 $e e$ 值可达 $86 \% \sim 90 \%$, 对 B 催化氢化的 ee 值最高也只有 $70 \%{ }^{[85]}$. 而配体 76c, 76d 以苯作溶剂对 $\mathbf{A}, \mathbf{B}$ 的催化氢化时间长 且效果差.<smiles>CC1(C)COC(=O)C1=O</smiles>

A<smiles>[B]C(=O)C(=O)NCc1ccccc1</smiles><smiles>[R2]OC(C)(C)CN([R2])[R2]</smiles>

75

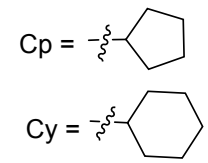

a: $R^{1}=R^{2}=C y$

b: $R^{1}=R^{2}=C p$

c: $R^{1}=C p, R^{2}=C y$

d: $R^{1}=C y, R^{2}=C p$

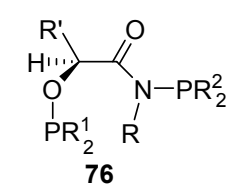

a: $\mathrm{R}=\mathrm{C}_{6} \mathrm{H}_{5} \mathrm{CH}_{2}, \mathrm{R}^{\prime}=\mathrm{Ph}, \mathrm{R}^{1}=\mathrm{Cp}, \mathrm{R}^{2}=\mathrm{Ph}$

b: $\mathrm{R}=\mathrm{C}_{6} \mathrm{H}_{5} \mathrm{CH}_{2}, \mathrm{R}^{\prime}=\mathrm{CH}_{3}, \mathrm{R}^{1}=\mathrm{Cp}, \mathrm{R}^{2}=\mathrm{Ph}$

c: $\mathrm{R}=\mathrm{C}_{6} \mathrm{H}_{5}, \mathrm{R}^{\prime}=\mathrm{C}_{6} \mathrm{H}_{5} \mathrm{CH}_{2}, \mathrm{R}^{1}=\mathrm{R}^{2}=\mathrm{Ph}$

d: $\mathrm{R}=\mathrm{C}_{6} \mathrm{H}_{5}, \mathrm{R}^{\prime}=\mathrm{CH}_{3}, \mathrm{R}^{1}=\mathrm{R}^{2}=\mathrm{Ph}$

图 9 配体 75 76 的结构

Figure 9 Structures of ligands $75 \sim 76$

Mortreux 课题组在 1988 年通过手性氨基酸设计合 成了新配体 77, 并将其与 $\mathrm{Ru}(\mathrm{II})$ 的配合物用于手性催化. 根据研究, 虽然 Ru(II)-77 催化氢化酮的效果一样, 但是 随着取代基的不同，与 $\mathrm{Ru}(\mathrm{I}), \mathrm{Ru}(\mathrm{II})$ 生成的配体对 $\mathbf{A}, \mathbf{B}$ 的 $e e$ 值只在 $40 \% \sim 85 \%$, 并且通过对比 $77 \mathbf{a} \sim 77 \mathrm{~b}$ 的催 化活性可以得到: $\mathrm{N}-\mathrm{P}$ 连接的基团部分可以控制反应 速度和立体选择, 还可以显著的增强催化剂的反应活性
和立体选择性. 同样来源的手性配体 78, 与 $\mathrm{Ru}$ 结合用 于 A 的手性催化氢化, ee 值可以达到 95\% 98\%, 但是 这个过程需要辅助配体. 考虑到电子效应的影响，该小 组进一步合成了配体 79, 实验证明 $\mathrm{Ru}-79 b$ 对 $\mathbf{A}$ 的催化 可以得到 $>90 \%$ 的 $e e$ 值. 这说明了电子效应对配体构型 的影响很大 ${ }^{[86]}$.

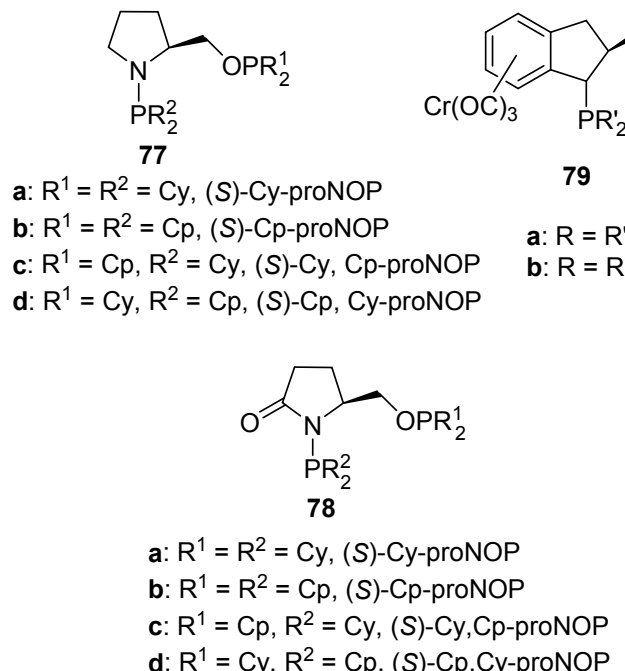

图 10 配体 77 79 的结构

Figure 10 Structures of ligands $77 \sim 79$

从糖类合成的配体，因糖具有多个手性中心，变化 繁多, 所以得到了化学家们的广泛关注. 自 1976 年 Kauabata 利用聚糖-纤维素为手性原料合成配体之后, 人们合成了大批单糖和双糖的手性膦配体如 80,81 (图 11), 在不对称催化反应中起到重要的作用. 到 1993 年 Bendayan 课题组再次合成了 4 个手性配体 $82 \sim 85$ 用于 $\mathbf{A}$ 的催化氢化上, 但是得到的转化率和 $e e$ 值都不是很 好, ee 值最大只能达到 20\%. 直到 2000 年 Mortreux 课题 组 ${ }^{[87]}$ 运用甘露糖醇的衍生物合成手性配体 86, 并将其 与 $\mathrm{Rh}$ 的配合物用于手性催化氢化, 当其辅助配体为 $\mathrm{CF}_{3} \mathrm{CO}_{2}{ }^{-}$时，可以得到 $80 \% \sim 90 \%$ 的 ee 值. 证实了这类 配体具有一定的局限性, 不是需要辅助配体, 就是立体 选择性不好.

\section{6 联芳环类手性膦配体}

具有阻转异构现象的手性联芳环类化合物在对映 选择性反应中是一类非常重要的手性配体，是在过渡金 属催化的不对称反应中最好的配体之一.

最早的联芳环类手性膦配体 87 是由 Tamao 课题 组 ${ }^{[88]}$ 合成报道的, 但是其不对称催化诱导性能很差. 直 到 1986 年 Noyori 等 ${ }^{[89]}$ 成功的合成了著名的 BINAP 双 膦配体 59, 此配体依赖连接两个萗环的 $\mathrm{C}-\mathrm{C}$ 单键旋转 


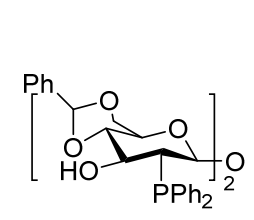

80

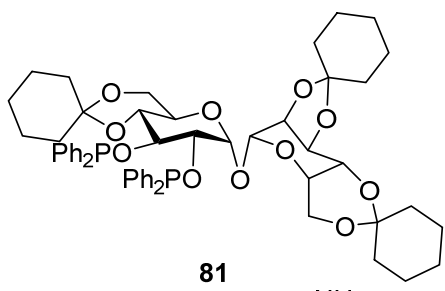

81<smiles></smiles>

$\operatorname{sh}^{2} \mathrm{PO} \mathrm{N}^{\mathrm{N}}$

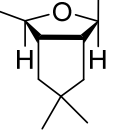

84

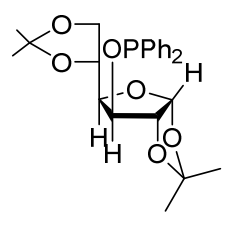

85<smiles>O=[PH](O)O[C@@H]1COC(c2ccccc2)[C@@H]([C@H]2OC(c3ccccc3)OC[C@H]2OP)O1</smiles>

86
图 11 配体 $80 \sim 86$ 的结构

Figure 11 Structures of ligands $80 \sim 86$

受阻而使整个分子具有旋光性.

在联荎类手性膦配体的研究中，一般是在芸环上引 入一些基团或者引入空间位阻很大的基团, 进而改变膦 配体的立体和电子效应. 如陈新滋课题组 ${ }^{[90]}$ 利用联䒺
酚的氧膦配体 88(图 12)研究了不对称氢氧化的催化性 能，其立体选择性可以达到 $92 \%$ 的 $e e$ 值. 在联萘类膦配 体中, 与氮原子相连的二苯基膦配体 (如 $\mathbf{8 9}^{[91]}, \mathbf{9 0}^{[92]}$ ), 还有引入配位能力的吡啶或噁唑啉的配体(如 $91^{[93]}$, $\mathbf{9 2}^{[94,95]}$ )在不同的反应中得到很好的选择性. 在 2000 年, 张绪穆课题组 ${ }^{[96]}$ 合成了配体 93, 并将其应用在 $\alpha, \beta$ 不饱 和酮的加氢反应中, 得到 $e e$ 为 $99 \%$ 的产物. 根据其方 法, 2006 年, Chan 小组 ${ }^{[97]}$ 开发了手性桥键连接的联苯类 配体 94, 在催化 $\alpha$-酮酯的不对称氢化反应中也表现出 很高的对映选择性, ee 值高达 $97 \%$. 2010 年, 张俊良课 题组 ${ }^{[98]}$ 以 94, 95 为手性配体, 实现了金催化的共轭烯炔 酮与硝酮的不对称加成反应，获得了优良的产率和立体 选择性，但对于环加成反应只能获得很低的对映选择 性, 直到 2014 年该课题 ${ }^{[99]}$ 组成功合成了配体 96, 才成 功地解决了催化环加成反应这类底物的问题. Lemaire 小组 ${ }^{[100]}$ 运用五步合成了光学纯的联菜骨架手性膦配体 97a 和 97b, 在 $\mathrm{Pd}$ 催化的苯乙烯的不对称硅氢化反应中 取得了较高的产率和对映选择性. 2005 年, 张绪穆课题 组 ${ }^{[101]}$ 根据配体 98 合成了配体 99, 将其用在催化甲酰基 化反应中, 可以得到 $99 \%$ 的 $e e$ 值.

联苯类双膦配体与联䒺类膦配体结构相似，也具有 优异的不对称催化诱导性能, 而且比联菜类膦配体更加

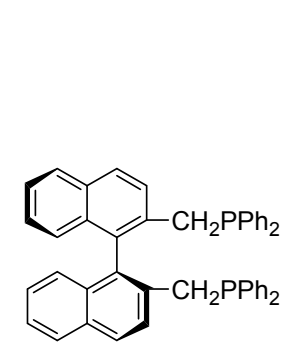

87

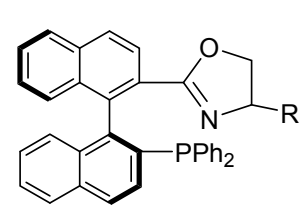

92

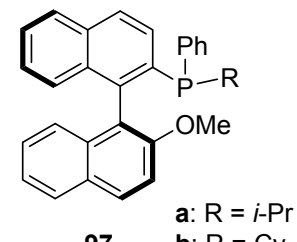

97

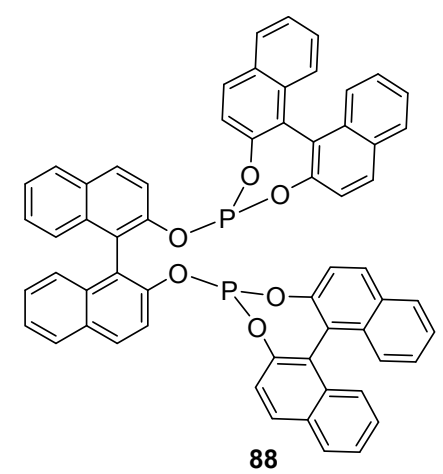

88

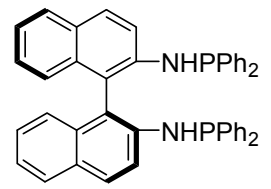

89

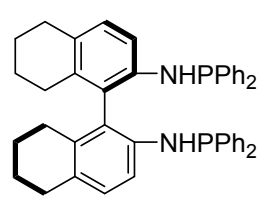

90

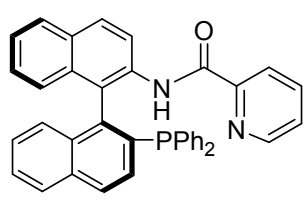

91

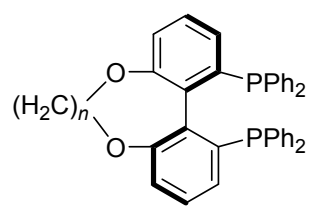

93<smiles>c1ccc2c(c1)OCOc1ccccc1-c1ccccc1-2</smiles>

94

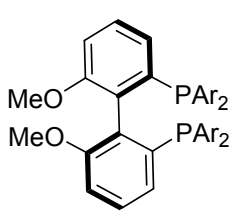

$\mathrm{Ar}=3,5-\left(t-\mathrm{C}_{4} \mathrm{H}_{9}\right)_{2}-4-\mathrm{MeOC}_{6} \mathrm{H}_{2}$

95

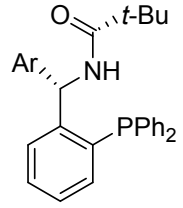

$\mathrm{Ar}=1-\mathrm{Naphthyl}$

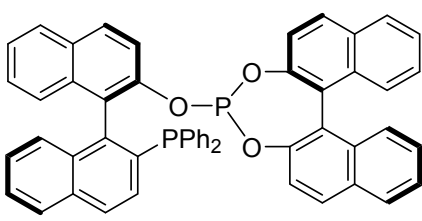

98

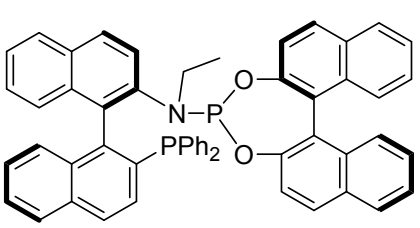

99

图 12 配体 87 99 的结构

Figure 12 Structures of ligands 87 99 
<smiles>Cc1cccc(-c2ccccc2)c1-c1c(C)cccc1-c1ccccc1</smiles>

100

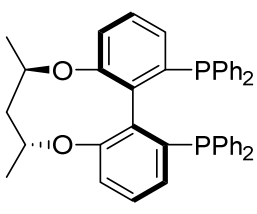

105<smiles>Cc1cc(C)c(-c2c(C)cc(C)cc2-c2ccccc2)c(-c2ccccc2)c1</smiles>

101

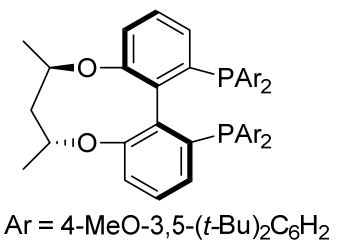

106
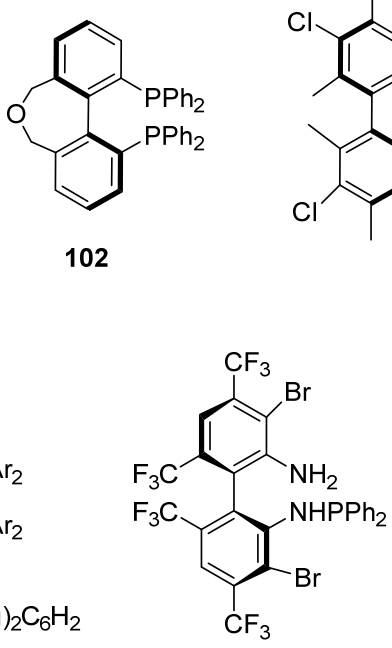

107

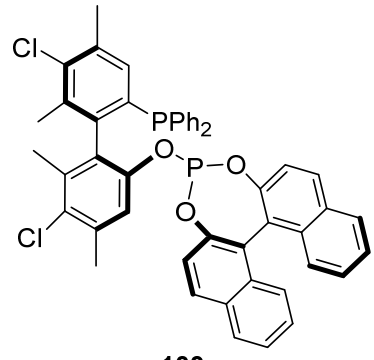

103
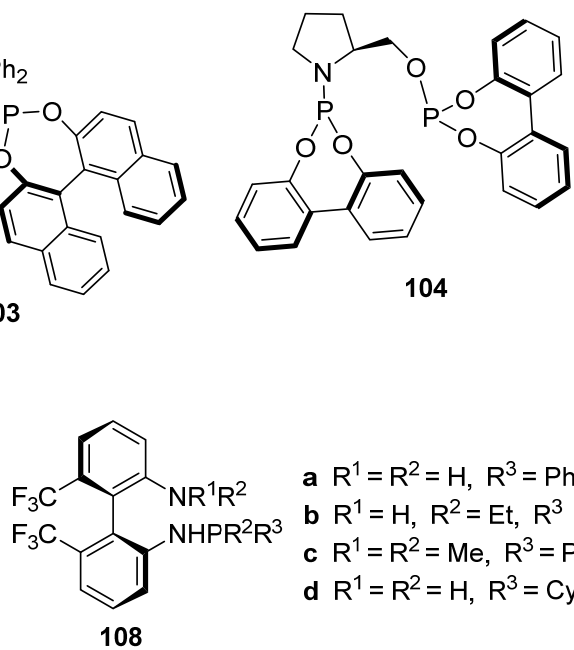

a $R^{1}=R^{2}=H, R^{3}=P h$ b $R^{1}=H, R^{2}=E t, R^{3}=P h$ c $\mathrm{R}^{1}=\mathrm{R}^{2}=\mathrm{Me}, \mathrm{R}^{3}=\mathrm{Ph}$ d $R^{1}=R^{2}=H, R^{3}=C y$

图 13 配体 $100 \sim 108$ 的结构

Figure 13 Structures of ligands $100 \sim 108$

易于修饰, 可引入位阻较大或者具有电子效应的基团. 这类化合物主要是通过乌尔曼偶联反应制得 ${ }^{[102]}$, 含有 不同吸电子基团和给电子基团的联苯膦配体得到广泛 的应用 (如配体 100 102) ${ }^{[103 ~ 109]}$. 联苯类氧膦配体 103, $104^{[103,104]}$ 可以用于不对称氢甲酰化反应, 并且具有一定 的立体选择性，该反应可以得到 30\% 35\%的ee 值, 实 验表明，当联苯类配体的磷原子上仅含有吸电子取代基 时, 反应活性则表现很差 ${ }^{[106]} .2008$ 年, 张绪穆课题 组 ${ }^{[110]}$ 合成了配体 $\mathbf{1 0 5}$, 在钓催化下的酮的加氢反应中, 配体 105 给出了 $92 \% e e$ 的对映选择性. 在此基础上, 王 春江课题组 ${ }^{[111]}$ 在 2013 年报道合成了配体 106, 在铱催 化下的芳香酮的加氢反应中, 配体 $\mathbf{1 0 5}$ 给出了 $99 \% e e$ 的对映选择性. 该课题组还在 2008 年合成了配体 $\mathbf{1 0 7}$, $\mathbf{1 0 8}^{[112]}$, 并在以后的七年中将其分别用在甲亚胺叶立德 和亚烷基丙二酸的不对称加成反应中 ${ }^{[13]}$ 、甲亚胺叶立 德和三氟烯烃的反应中 ${ }^{[14]}$ 、甲亚胺叶立德和丁内脂的 反应 ${ }^{[15]}$ 和用甲亚胺叶立德合成吡咯衍生物的反应 中 $^{[116]}$, 分别得到 $65 \%, 93 \%, 98 \%, 97 \%$ 的 $e e$ 值.

随着联芸和联苯类膦配体的成功应用, 人们认识到 具有 $C_{2}$ 对称轴的阻转异构膦配体很有研究价值, 开始 将研究转移到联杂环膦配体的合成上. 联杂环类配体包 括对称的即同一杂环偶联而成的膦配体及一分子杂环 和苯环或菜环偶联而成的手性膦配体. 1995 年 Benincori 等 ${ }^{[117,118]}$ 最早报道了带取代基的联苯并噻吩手性配体 109, 这种配体中磷原子的电子密度有所增强, 这样就 增强了与过渡金属的配位能力, 对 $\beta$-酮酸酯催化还原 时, 可以得到 $99 \%$ 的 $e e$ 值. 通过这样的方式, 该课题组 ${ }^{[119]}$ 又合成了 $\mathrm{N}-\mathrm{N}$ 键连的联苯哒唑膦配体和其他杂环
类的手性配体 110 112. 将配体 111、112 用于 Heck 反 应中可以得到 $e e$ 值 $(86 \% \sim 96 \%)$ 和产率 $(76 \% \sim 93 \%)$ 均高 于 BINAP 催化诱导的结果 ${ }^{[120]}$. Brown 等 ${ }^{[121]}$ 较早报道了 联吲哚双膦配体 113 的合成, 但是却没有应用于不对称 催化的研究报道. 1999 年 Hiemstra 等 ${ }^{[119]}$ 报道了二苯并 呋喃及其衍生物的手性配体 114 116, 但是其在不对称 催化氢化过程中相比 BINAP 需要的反应时间较长. 与 此同时, Guiry 等 ${ }^{[122]}$ 合成了含吡嗪环的芸单膦配体 117, 但是因吡嗪环太小，长时间难以避免分子的消旋化. 同 时合成了含有喹唑啉环和萗环的新配体 118, 在不对称 烷基化反应中具有很高的转化率, 但只有中等的 $e e$ 值, 且在不对称硼氢化反应过程中的立体选择性也不是很 好 ${ }^{[122]}$.

在这些配体中, 由于 $\mathrm{N}, \mathrm{O}, \mathrm{S}$ 等杂原子的参与, 可以 调节其电子性能, 并能较方便地控制配位数和立体构 型，提高配合物的刚性，因而在不对称催化中具有较高 的光学选择性.

\section{7 含二茂铁基的手性膦配体}

在不对称催化研究中, 具有面手性的二茂铁类配体 越来越受到重视. 自 1970 年 Ugi 等 ${ }^{[123]}$ 成功地将 $\alpha-N, N$ 二甲基二茂铁乙胺拆分以来, 已合成了数以百计的 $\alpha$-手 性二茂铁衍生物 ${ }^{[124]}$, 这些配体已经成功的运用到各种 不对称反应中. 戴立信团队 ${ }^{[125]}$ 也设计合成了一系列二 茂铁膦配体, 取得了令人瞩目的成果. Mathey 课题 组 ${ }^{[126]}$ 研究合成了一种二茂铁类似物的膦配体 119, 并将 其运用到不对称催化过程中, 得到了很好的结果. 早期, Hayashi 等 ${ }^{[127]}$ 利用磷杂茂合成了一系列手性二茂铁膦配 


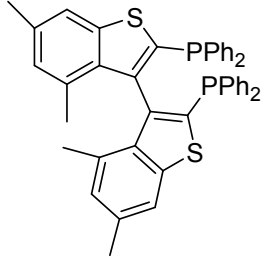

109<smiles>c1ccc(-c2nc3ccccc3n2-c2nc3ccccc3n2-c2ccccc2)cc1</smiles>

110<smiles></smiles>

111<smiles>Cc1sc(C)c(-c2c(C)sc(C)c2-c2ccccc2)c1-c1ccccc1</smiles>

112<smiles>Pc1ccccc1-c1nc(-c2ccccc2)c(-c2ccccc2)[nH]1</smiles>

113

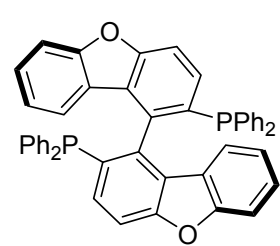

114

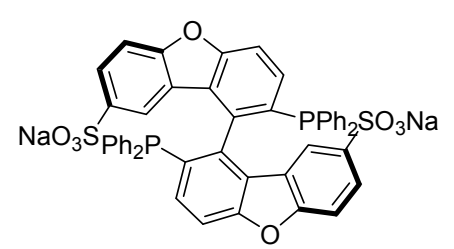

115

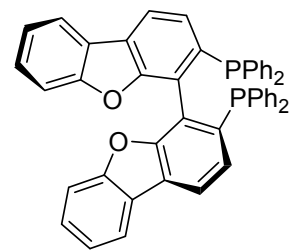

116

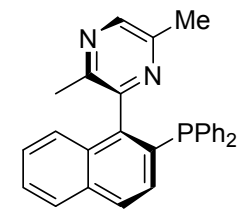

117<smiles>Cc1nc(-c2c(-c3ccccc3)ccc3ccccc23)c2ccccc2n1</smiles>

118

图 14 配体 109 118 的结构

Figure 14 Structures of ligands $109 \sim 118$

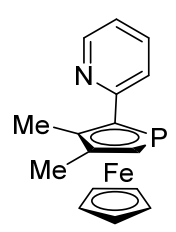

119

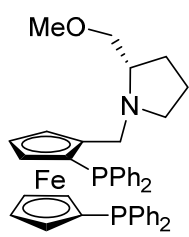

126

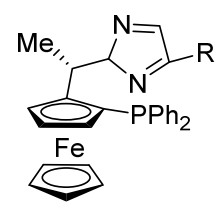

127

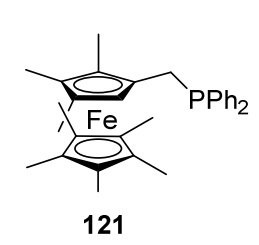

121

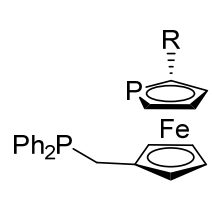

122

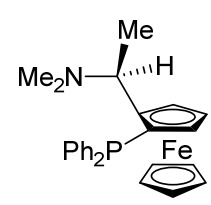

123

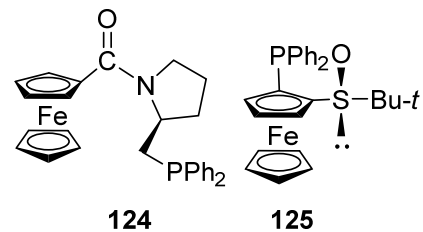

125<smiles>c1ccc(Pc2cc3c(c(-c4ccccc4)c2)CCCC3)cc1</smiles>

128<smiles></smiles>

129<smiles>CC(NC(=S)Nc1cc(C(F)(F)F)cc(C(F)(F)F)c1)C1C=CCC(C2C=CC2Pc2ccccc2)C1P</smiles>

130

图 15 配体 119 130 的结构

Figure 15 Structures of ligands $119 \sim 130$

体 $120 \sim 123$, 并考查了它们在不对称烯丙基化反应的 催化诱导性能, 均表现出很好的立体选择性, ee 值高达 $99 \%{ }^{[128]}$. 2005 年, 王敏灿课题组 ${ }^{[129]}$ 合成了手性二茂铁 基配体 124, 将其用于铜催化的二乙基锌和亚胺的不对 称加成反应中, 可以得到高于 $88 \% e e$ 值. 2012 年, 我们 课题组李高伟 ${ }^{[130]}$ 合成了具有平面手性和中心手性的二 茂铁基亚砜膦配体 125, 将其用于催化 1,3-二苯基-2-丙 烯基乙酸酯和丙二酸二甲酯/氢化钠的模板反应, 只能 得到 $20 \%$ 的 $e e$ 值. 2013 , 我们课题组 ${ }^{[131]}$ 又报道了通过 “一锅煮” 的方法合成了一种新型的二茂铁基手性双膦 配体 126, 该配体在不对称催化中的应用还在进一步研 究中.

在二茂铁的茂环上引入含氮杂环(如配体 $127^{[132]}$ )或 者引入较强刚性的环己烷(如配体 $\mathbf{1 2 8}^{[133]}$ ) 也可以在不同 的不对称氢化反应中取得很好的结果. 2003 年, 张绪穆
课题组 ${ }^{[134]}$ 合成了具有联䒺的二茂铁配体 129, 在铱催化 下, 在酮的催化加氢反应和烯胺的还原反应中, 配体 128 分别给出了 $95 \%$ ee 和 $94 \%$ ee 的对映选择性. 在同 一年, 该课题组 ${ }^{[135]}$ 还合成报道了含有二茂铁的手性膦 硫脲配体 130, 并将其应用在催化 $\beta, \beta$-取代硝基烯烃的 选择性加氢反应中最高可以达到 $99 \%$ 的产率和 $99 \%$ 的 对映选择性; 在铑催化下, 合成了手性 $\beta$ 氨基硝基烷烃 产物及其衍生物, 得到的产物 ee 高达 96\%, 产率为 $97 \%{ }^{[136]}$. 有关二茂铁膦配体的综述已经有报道 ${ }^{[137]}$. 这 里不再过多描述.

\section{8 其他手性膦配体}

除上述的各种膦配体外, 同样还有许多其他膦配体 在不对称催化反应中表现出非常好的催化性能, 如合成 的一些特殊的配体 131 135 ${ }^{[138 ~ 143]}$, 其中有些是由多元 
<smiles>Oc1c(P)cccc1P(O)c1ccccc1/C=N\[C@H]1c2ccccc2/C=N\[C@H](c2ccccc2)c2ccccc21</smiles>

131<smiles>PC1=CC=CC=CC(C(Cc2ccccc2)c2ccccc2)=C1P</smiles>

132<smiles>CC1C(c2ccccc2)OP(Oc2cccc3cccnc23)N1[Na]</smiles>

133

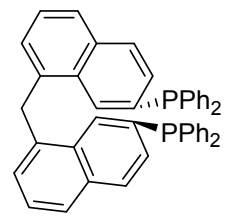

134<smiles>CCOCC1OP(OC2C(C)C3(C)CCC2(C)C3(C)C)OC1C(OCC)OCC</smiles><smiles>O=P1(c2ccccc2O)CN(c2ccccc2)[C@@H]2CCCN21</smiles>

136<smiles>O=P1(c2ccc3ccccc3c2O)N(c2ccccc2)C[C@@H]2CCCN21</smiles>

137<smiles>[R]C(C)(C)OP(=O)(Oc1ccc2ccccc2c1)Oc1ccc2ccccc2c1-c1ccccc1</smiles>

138<smiles>CCOc1cccc(CC(C)(C)Oc2cccc(CC(C)(C)Oc3cccc(C(C)(C)Cc4ccccc4)c3)c2)c1</smiles><smiles>O=CNc1cccc2c(-c3c(P)ccc4ccccc34)c(-c3ccccc3)ccc12</smiles><smiles>CCOc1cccc(CCOc2cccc(CC(C)(c3ccccc3)c3ccccc3)c2)c1</smiles>

139

图 16 配体 131 139 的结构

Figure 16 Structures of ligands 131 $\sim 139$

稠环或刚性很强的环得到的. 科学家一般致力于三价膦 配体的研究, 而 Buono 课题组 ${ }^{[144]}$ 合成了五价磷的配体 136，137，运用在苯甲酫与二乙基锌加成反应中，分别 得到了 $73 \%$ 和 $98 \%$ 的 $e e$ 值. Inanaga 等 ${ }^{[145]}$ 合成的联䒺氧 膦 $(\mathrm{V})$ 金属复合物也可以看作是由一种五价膦配体 $\mathbf{1 3 8}$ 得到的催化剂, 该催化剂用在烯酮的甲氧氢氨的 1,4 加 成反应中, 得到很好的诱导性能, 其钪(Sc)复合物的不 对称催化可达 $84 \% e e$ 值. 2000 年, 范青华课题组首次报 道了以手性膦配体为核心的树状大分子膦配体 139 , 目 前已经将其发展成为系列手性树状分子, 并将其与铑、 钓和铱形成配合物, 成功地应用到烯烃、酮和亚胺(喹 啉)等底物的不对称氢化反应中. 虽然反应中可以看到 树状分子的效应, 但是树状分子如何影响催化剂的活性 以及选择性, 目前还不是很清楚 ${ }^{[146]}$.

\section{9 手性膦配体的发展趋势}

合成手性膦配体的目的就是要把它制备成催化剂 用于不对称催化合成反应中 ${ }^{[147]}$, 纵观近年来的手性膦 配体的合成研究, 主要是配体的改进和创新. 具体的研 究有以下几个方面.

\section{1 手性膦配体的改进}

Hayashi 等 ${ }^{[148]}$ 对 BPPFA 配体进行改进合成了一种 新的配体 140, 用在对取代烯烃的不对称加氢上, 因其
起修饰作用的基团参与了金属的配位，所以取得了

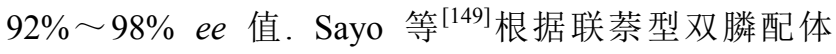
BINAP 合成了联苯型双膦配体 BIPHEN 100, 其在各种 溶剂中的溶解性均优于 BINAP, 在一些不对称氢化反应 中更是优于 BINAP 的立体选择性.

\section{2 水溶性手性膦配体的合成}

水溶性手性配体的合成, 主要是为了分离催化剂和 产物，从而使手性配体和金属有可能得到重复循环使 用. 膦的水溶性化合物的获得是通过一个强极性官能团 的引入, 例如配体 141，142 ${ }^{[150,151]}$. Ishizaki 等 ${ }^{[152]}$ 利用磺 酸基制备成水溶性膦配体 141, 由配体形成的络合物可 以在水溶液中进行, 反应条件温和，从而使该体系的应 用范围得到进一步扩大.

\section{3 负载型手性膦配体的合成}

负载型手性膦配体的出现, 也是为了解决催化剂和 产物的分离. 主要是将催化剂负载与载体上, 而载体可 以是有机高分子, 也可以是无机物. 负载型手性配体的 合成主要有以下两条途径: 一是在高聚物的载体中引入 活泼的基团, 使可以与手性配体反应, 得到含有手性膦 支链的聚合物 ${ }^{[153 ~ 155]}$, 如 143; 二是制得含有双键的手 性膦配体作为单体, 然后再进行聚合或者与其他单体共 聚, 得到负载型手性配体. 如 Stille ${ }^{[156]}$ 合成的聚合物固 载手性双膦配体 144, 在不对称氢甲酰化反应中得到 
$73 \%$ 的 $e e$ 值. 易兵等 ${ }^{[157]}$ 合成了咪唑型负载手性双膦配 体 145, 并用于 $\alpha$-乙酰氨基肉桂酸的不对称氢化反应, 产物的转化率为 $100 \%$, 对映选择性为 $97 \%$.

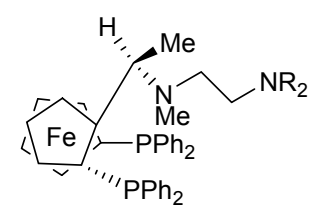

140<smiles>O=C(O)c1cc(C(=O)O)cc(C(=O)N2C[C@@H](P)CC2CPc2ccccc2)c1</smiles>

142

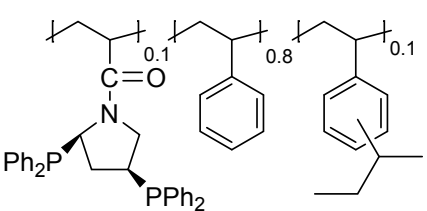<smiles>Cn1cc[n+](CC(=O)N2C[C@H](Pc3ccccc3)[C@H](P(c3ccccc3)c3ccccc3)C2)c1</smiles>

144

145<smiles>CS(=O)(=O)c1cccc(-c2c(-c3c(P(c4cccc(S(C)(=O)=O)c4)(c4cccc(S(C)(=O)=O)c4)(c4cccc(S(C)(=O)=O)c4)c4cccc(S(C)(=O)=O)c4)ccc4ccccc34)ccc3ccccc23)c1</smiles>

图 17 配体 140 146 的结构

Figure 17 Structures of ligands $140 \sim 146$

\section{4 负载型的水溶性配体的合成}

1994 年, Wan 和 Davis ${ }^{[158]}$ 根据水溶性手性膦配体和 负载型手性膦配体的不同特点合成了一种新型负载型 的水溶性催化剂体系. 这种催化剂含有一个水溶性的有 机金属络合物, 这种有机金属络合物包含在一个负载在 一种高表面的亲水性固体的水膜里, 而反应物和产物则 在一个疏水性的有机相中. 它的催化剂组成为 146, 用 于不对称加氢反应中 $e e$ 值达到 $96 \%$.

\section{5 手性环状胺膦配体的合成}

手性胺膦配体因同时含有 “软” 的磷原子和 “硬” 的氮原子而具有丰富的配位化学性能和优秀的不对称 诱导能力. 该配体中磷原子具有 $\pi$ 受体的特性, 可以增 加金属中心离子的碱性和提高配合物的稳定性. 而氮原
子因为具有 $\sigma$ 电子给予性能可以与中心金属原子生成较 弱的配位键. 李岩云课题组 ${ }^{[159 ~ 163]}$ 自行设计合成了一系 列手性环状胺膦配体 $147 \sim 152$, 这些配体具有丰富的 配位化学性能, 分别与不同的过渡金属生成的催化体系 在多种类型的不对称催化反应中显示了独特的对映选 择性. 将这些膦配体用在酮的不对称还原中都可以得到 大于 $90 \%$ 的 $e e$ 值. 2008 年, Trost 课题组 ${ }^{[164]}$ 合成报道了 配体 152, 并将其用在不对称烯丙基烷基化反应中，得 到很好的对映选择性.

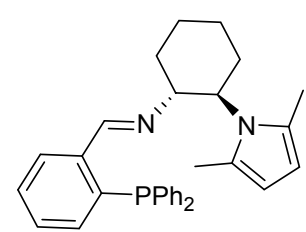

147<smiles>Pc1ccccc1/C=N/C(c1ccccc1)c1ccccc1Pc1ccccc1</smiles>

149
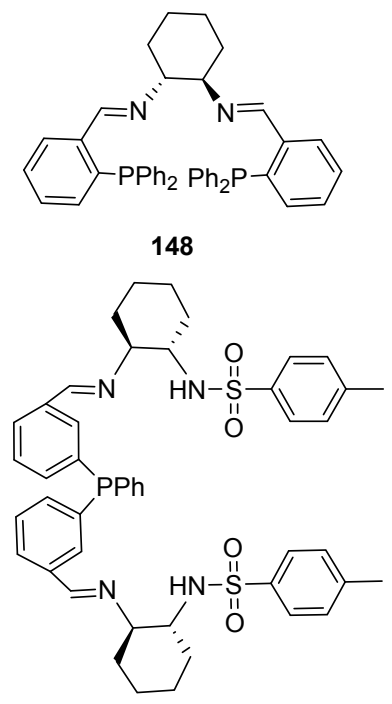

150<smiles>CC(C)(C)OC(=O)N[C@H]1CCCC[C@@H]1N=Cc1cccc(P(c2ccccc2)c2cccc(C=N[C@@H]3CCCC[C@H]3NC(=O)OC(C)(C)C)c2)c1</smiles>

151

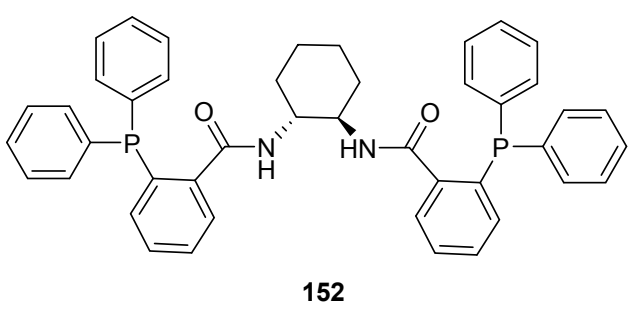

图 18 配体 147 152 的结构

Figure 18 Structures of ligands $147 \sim 152$

\section{6 具有螺环骨架的手性膦配体}

从 20 世纪 90 年代, 特别是进入 21 世纪以来, 具有 螺环骨架的手性膦(磷)配体受到了广泛的关注, 并逐渐 发展成为特色鲜明的手性配体类别. 由陈新滋、蒋耀忠、 张绪穆、周其林、丁奎岭等著名有机化学家, 将手性螺 
环配体的骨架由多手性的螺[4.4]壬烷骨架发展到只具 有单一手性的螺二氢狮和螺 [4.4]壬二烯等螺环骨架类 型, 形成了包括手性螺环单磷配体、双膦配体、膦氮配 体等丰富的手性配体库 ${ }^{[165 ~ 168]}$. 如 153 164. 这些手性 螺环膦配体及其催化剂不仅在不对称催化氢化、不对称 碳一碳键形成、不对称碳一杂原子键形成等多种类型的不 对称催化反应中均表现出优异的催化活性和对映选择 性, 而且使得许多原先难以控制对映选择性的不对称催 化反应变得可能. 而今, 手性螺环结构已成为 “优势结 构”，相应的手性螺环膦配体及其催化剂已被国内外同 行广泛采用. 手性螺环膦配体的兴起为手性催化剂研究 增加了活力, 极大地促进了不对称合成化学的发展. 今 后, 手性螺环膦配体的研究除了将向新型、高效、高选 择性手性配体及催化剂方向发展外, 将其应用于新的不

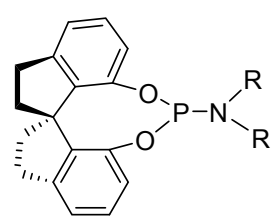

153 SIPHOS<smiles>Pc1ccccc1PN[C@@H]1CCCC12CCCC2</smiles>

156 SpiroNP

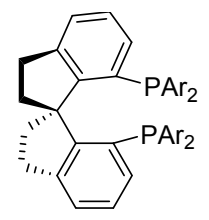

154 SDP

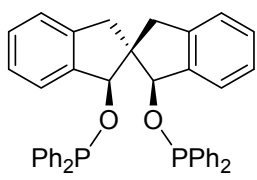

157 SpiroBIP

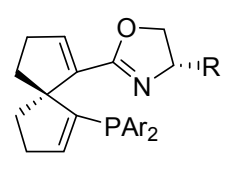

155 SpinPHOX

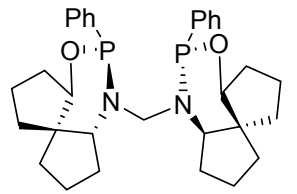

159

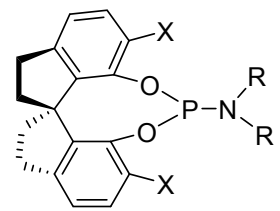

161

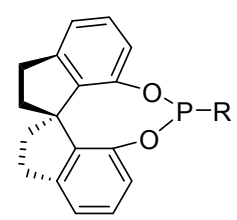

163 FuP

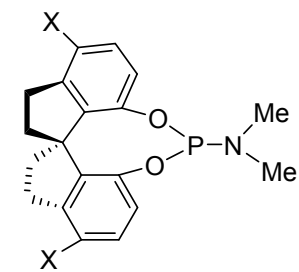

160

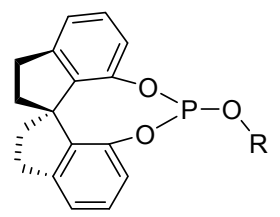

162 ShiP

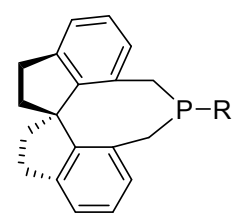

164 SITCP

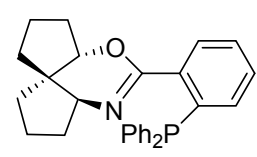

158
对称催化反应的对映选择性控制、以及应用于手性天然 产物和药物的高效不对称合成将成为新的研究热点.

\section{0 结论与展望}

手性膦配体在不对称催化反应中已经得到了非常 广泛的应用, 但是在不对称催化氢化等领域仍然存在很 多问题和挑战. (1)对于不含附属功能基底物的氢化，大 多数催化剂都不能获得较高的对映选择性, 有时甚至难 以使反应进行. (2)新型的手性催化剂的合成还应该是在 改变催化剂体系的结构和组成上, 以达到提高对映选择 性和催化效率的目的. (3)多数的手性膦配体催化的反应 中都不可避免地使用了贵金属，如钯、铑、铱、金、钌 等作为催化剂, 如何开发廉价低毒的金属催化体系以及 非金属催化体系, 提高反应的原子转化数(TON)等方面 也将是其面临的挑战之一. (4)在未来的不对称催化反应 研究中, 寻找和发现新颖配体骨架并开展新型高效的手 性膦配体及催化剂的设计合成，仍是不对称催化反应研 究的核心内容之一, 并将继续是人们关注的热点. (5)目 前，手性膦配体参与的不对称催化反应还缺乏成体系的 理论指导, 相关的动力学研究以及对反应历程的理解方 面还有待进一步的深入研究. (6)绝大多数优秀高效的手 性膦配体的合成都比较困难，如何解决手性配体的回收 和循环使用这一科学问题, 仍将是化学家面临的挑战之

\section{References}

[1] Knowles, W. S.; Sabacky, M. J. J. Chem. Soc., Chem. Commun. 1968, 20, 1445.

[2] Horner, L.; Sieged, H.; Buthe, H. Angew. Chem., Int. Ed. Engl. 1968, 7, 942.

[3] Knowles, W. S.; Sabacky, M. J. US 3849480, 1968 [Chem. Abstr. 1971, 82, 86086].

[4] William, H. P.; Minn, C. C. J. Org. Chem. 1987, 52, 3176

[5] Noyori, R. Science 1990, 248, 1194.

[6] Parshall, G. W.; Nugent, W. A. CHEMTECH 1988, 18, 376.

[7] Hayashi, T.; Katsumura, A.; Konishi, M.; Kumada, M. Tetrahedron Lett. 1979, 20, 425.

[8] Kagan, H. B. Adv. Catal. 1986, 25, 88.

[9] Uozumi, Y.; Hayashi, T. J. Am. Chem. Soc. 1991, 113, 9887.

[10] Kitayama, K.; Tsuji, H.; Uozumi, Y.; Hayashi, T. Tetrahedron Lett. 1996, 37, 4169.

[11] Farkas, G.; Balogh, S.; Szollosy, A.; Uerge, L.; Darvas, F.; Bakos, J. Tetrahedron: Asymmetry 2011, 22, 2104.

[12] Urbaneja, X.; Mercier, A.; Besnard, C.; Kündig, E. P. Chem. Commun. 2011, 47, 3739.

[13] Li, W. G.; Zhang, Z. G.; Xiao, D. M.; Zhang, X. M. J. Org. Chem. 2000, 65, 3489.

[14] Zhang, W. C.; Zhang, X. M. J. Org. Chem. 2007, 72, 1020.

[15] (a) He, H.; Zheng. X. J.; You, S. L. Org. Lett. 2007, 9, 4339. (b) Liu, W. B.; He, H.; You, S. L. Org. Lett. 2008, 10, 1815. (c) Xu, Q. L.; Dai, L. X.; You, S. L. Org. Lett. 2009, 12, 800. (d) Xu, Q. L.; Dai, L. X.; You, S. L. Org. Lett. 2012, 14, 2579. 
[16] (a) Xu, Q. L.; Liu, Q. L.; Dai, L. X.; You, S. L. J. Org. Chem. 2010, 75,4615 .

(b) Wu, Q. F.; He, H.; You, S. L.; Liu, W. B. J. Am. Chem. Soc. 2010, 132, 11418 .

(c) Zhou, C. X.; Zheng, C.; You, S. L. Acc. Chem. Res. 2014, 47, 2558

(d) Yang, Z. P.; Wu, Q. F.; You, S. L. J. Am. Chem. Soc. 2015, 135, 15899.

[17] Li, K.; Hu, N.; Luo, R. S.; Yuan, W. C.; Tang, W. J. J. Org. Chem. 2013, 78, 6350 .

[18] (a) Yang, X. T.; Xu, G. Q.; Tang, W. J. Tetrahedron 2016, 72, 5178. (b) Du, K.; Guo, P.; Chen, Y.; Tang, W. J. Angew. Chem., Int. Ed. 2015, 54, 3033 .

[19] Hu, N. F.; Li, K.; Wang, Z.; Tang, W. J. Angew. Chem., Int. Ed. 2016, 55,1 .

[20] Li, Y. M.; Fan, Q. H.; Chen, X. Z. Asymmetric Organic Reaction, Chemical Industry Press, Beijing, 2005 (in Chinese). (李月明, 范青华, 陈新滋, 不对称有机反应, 化学化工出版社, 北京, 2005.)

[21] Burk, M. J.; Tharper, G. P.; Christopher, S. J. Am. Chem. Soc. 1995, $117,4423$.

[22] Imamoto, T.; Watanabe, T.; Wada, Y.; Masuda, H.;Yamada, H.; Tsuruta, H.; Matsukawa, S.; Yamaguchi, K. J. Am. Chem. Soc. 1998, 120, 1635.

[23] Imamoto, T.; Tamura, K.; Zhang, Z. F.; Horiuchi, Y. Sugiya, M.; Yoshida, K.; Yanagisawa, A.; Gridnev, L. D. J. Am. Chem. Soc. 2011, 133, 1754.

[24] Zhang, Z.; Tamura, K.; Mayama, D.; Sugiya, M.; Imamoto, T. J. Org. Chem. 2012, 77, 4184.

[25] (a) Geng, H. L.; Huang, K. X.; Sun, T.; Li, W.; Zhang, X. W.; Wu, W. J.; Zhang. X. M. J. Org. Chem. 2011, 76, 332.

(b) Huang, K. X.; Zhang, X. W.; Geng. H. L.; Li, S. K.; Zhang, X. M. ACS Catal. 2012, 2, 1343.

(c) Jiang, J.; Liu, W. X.; Lv, H.; Zhang, X. M. Org. Lett. 2015, 17, 1154 .

[26] Tang, W. J.; Qu, B.; Andrew, G.; Chris, H.; Rodriguez, S.; Wei, X. D.; Haddad, N.; Narayanan, B.; Ma, S. L. Org. Lett. 2010, 12, 176.

[27] (a) Liu, G. D.; Liu, X. Q.; Cai, Z. H.; Tang, W. J. Angew. Chem., Int Ed. 2013, 52, 4235 .

(b) Luo, R. S.; Li, K.; Hu, Y. L.; Jiao, G. J.; Xu, G. P.; Tang, W. J. Adv. Synth. Catal. 2013, 355, 1297.

[28] Huang, L. W.; Zhu, J. B.; Jiao, G. J.; Wang, Z.; Yu, X. X.; Deng, W. P.; Tang, W. J. Angew. Chem., Int. Ed. 2016, 55, 4527.

[29] Morrison, J. D.; Masler, W. F. J. Org. Chem. 1974, 39, 270.

[30] Morison, J. D.; Burnett, R. E. Aguiar, A. M.; Morrow, C. J.; Phillips, C. J. Am. Chem. Soc. 1971, 93, 1301.

[31] Dawson, G. J.; Frost, C. G.; Williams, J. M. J.; Coote, S. J. Tetrahedron Lett. 1993, 34, 3149.

[32] Sprinz, J.; Helmechen, G. Tetrahedron Lett. 1993, 34, 1769

[33] Janssen, J. P.; Helmechen, G. Tetrahedron Lett. 1997, 38, 8025.

[34] Yang, S. X.; Chen, R. R.; Li, R. G. Chin. J. Org. Chem. 1980, (4), 1 (in Chinese).

(杨石先，陈茹玉，李玉桂，有机化学, 1980, (4), 1.)

[35] Pasquier, C.; Naili, S.; Pelinski, L.; Brocard, J.; Mortreux, A.; Agbossou, F. Tetrahedron: Asymmetry 1998, 9, 193.

[36] Hamada, Y.; Seto, N.; Ohmori, H.; Hatano, K. Tetrahedron Lett. 1996, 37, 7565

[37] Chen, Z. G.; Jiang, Q. Z.; Zhu, G. X. ;Xiao, D. M.; Cao, P.; Guo, C.; Zhang, X. M. J. Org. Chem. 1997, 62, 4521.

[38] Zhu, G. X.; Chen, Z. G.; Jiang, Q. Z. J. Am. Chem. Soc. 1997, 119, 3836.

[39] Miyashita, A.; Yasuda, A.; Takaya, H.; Toriumi, K.; Ito, T.; Noyori,
R. J. Am. Chem. Soc. 1980, 102, 7932.

[40] Yang, H.; Alvarez-Gressier, M.; Lugan, N.; Mathieu, R. Organometallics 1997, 16, 1401.

[41] Okda, T.; Morito, T.; Achiwa, K. Chem. Lett. 1990, 16, 999.

[42] Zhao, J.; Gu, R. J. Chem. Res. 1997, 8, 35 (in Chinese). (赵瑾, 谷如驹, 化学研究, 1997, 8, 35.)

[43] Hayashi, T.; Konishi, M.; Fukushima, M. Bull. Soc. Chim. Belg. 1979, 11, 923

[44] Meek, D. W. In Homogeneous Catalysis with Metal Phosphine Complexes, Plenum Press, New York, 1983, Chapter 8.

[45] Dang, T. P.; Kagan, H. B. J. Chem. Soc. 1971, 93, 484;

[46] Henri, B. K. J. Am. Chem. Soc. 1972, 94, 6429.

[47] Brown, J. M.; Murrer, B. A. Tetrahedron Lett. 1980, 21, 581.

[48] Towsend, J. M.; Blount, J. F.; Sun, R. C.; Zawoiski, S.; ValentineJr, D. J. Org. Chem. 1980, 45, 2995.

[49] Yamagishi, T.; Yatagai, M.; Hatakeyama, H.; Hida, M. Bull. Chem. Soc. Jpn. 1984, 57, 1897.

[50] Morimoto, T.; Chiba, M.; Achiwa, K. Tetrahedron 1993, 49, 1793.

[51] Tao, X. M.; Li, W. F.; Ma, X.; Li, X. M.; Zhu, L. F.; Xie, X. M. Zhang, Z. G. J. Org. Chem. 2012, 77, 8401.

[52] Burk, M. J.; Feaster, J. E.; Nugent, W. A.; Harlow, R. L. J. Am. Chem. Soc. 1993, 115, 10125.

[53] Pye, P. J.; Rossen, K.; Reamer, R. A.; Tsou, N. N.; Volante, R. P.; Reider, P. J. J. Am. Chem. Soc. 1997, 119, 6207.

[54] Burk, M. J. J. Am. Chem. Soc. 1991, 113, 8518.

[55] Burk, M. J.; Feaster, J. E.; Harlow, R. L. Organometallics 1990, 9 , 2653.

[56] Burk, M. J. J. Am. Chem. Soc. 1991, 113, 8518.

[57] Burk, M. J.; Feaster, J. E.; Nugent, W. A. J. Am. Chem. Soc. 1993, 115,10125 .

[58] Burk, M. J.; Lee, J. R.; Martinez, J. P. J. Am. Chem. Soc. 1994, 116, 10847.

[59] (a) Achiwa, K. J. Am. Chem. Soc. 1976, $98,8265$. (b) Stille, J. K. Chem. Ind. 1985, 22, 23.

[60] Baker, G. L.; Fritschel, S. J.; Stille, J. R.; Stille, J. K. J. Org. Chem. 1981, 46, 2954.

[61] Takahashi, H.; Hattori, M.; Chiba, M.; Chiwa, A.; Morimoto, T. Tetrahedron Lett. 1986, 27, 4477.

[62] RajanBabu, T. V.; Ayers, T. A.; Halliday, G. A.; You, K. K.; Calabrese, J. C. J. Org. Chem. 1997, 62, 6012.

[63] Habus, I.; Raza, Z.; Sunjic, V. J. Mol. Catal. 1987, 42, 173.

[64] Fryzuk, M. D.; Bosnich, B. J. Am. Chem. Soc. 1977, 99, 6262.

[65] Behar, D.; Neta, P. J. Am. Chem. Soc. 1981, 103, 2280.

[66] Miyashita, A.; Yasuda, A.; Takaya, H.; Toriumi, K.; Ito, T.; Noyori, R. J. Am. Chem. Soc. 1980, 102, 7932.

[67] Miyashita, A.; Takaya, H.; Souchi, T.; Noyori, R. Tetrahedron 1984, 40, 1245.

[68] Takaya, H.; Mashima, K.; Koyano, K.; Yagi, M.; Kumobayashi, H.; Taketomi, T.; Akutagawa, S.; Noyori, R. J. Org. Chem. 1986, 51, 629.

[69] Takaya, H.; Akutagawa, S.; Noyori, R. Org. Synth. 1988, 67, 20.

[70] Burk, M. J. J. Am. Chem. Soc. 1991, 113, 8518.

[71] Jiang, Q.; Jiang, Y.; Xiao, D. M.; Zhang, X. Angew. Chem., Int. Ed. Engl. 1998, 37, 1100

[72] Kyba, E. P.; Sandoval, C. A.; Yamaguchi, Y.; Zhang, X.; Wang, Z.; Kato, K.; Liu, S. T.; Harris, R. L. Organometallics 1983, 2, 1877.

[73] (a) Liu, Y.; Ding, K. L. J. Am. Chem. Soc. 2005, 127, 10488. (b) Yan Liu, Christian, A.; Ding, K. L. J. Am. Chem. Soc. 2006, 128,14212

[74] Schmid, R.; Broger, E. A.; Cereghetti, M.; Crameri,Y.; Foricher, J Pure Appl. Chem. 1996, 68, 131.

[75] Philip, J. P.; Rossen, K.; Robert, A. R.; Tsou, N. N.; Volante, R. P.; 
Reider, P. J. J. Am. Chem. Soc. 1997, 119, 6207.

[76] Brenchley, G.; Erifield, E.; Wills, M.; Fedouloff, M. Tetrahedron Lett, 1994, 35, 2791.

[77] Sennlenn, P.; Gabler, B.; Helmchen, W. Tetrahedron Lett. 1994, 35, 8595.

[78] Whitesell, J. K. Chem. Rev. 1989, 89, 1581.

[79] Kumar, A.; Srivastava, N.; Mital, A. J. Chem. Soc., Chem. Commun. 1992, 32, 493.

[80] Jing, Y. Z.; Mi, A.; Yan, M.; Sun, J.; Lou, R. L.; Deng, L. G. J. Am. Chem. Soc. 1997, 119, 9570.

[81] Chan, A.; Jiang, S. C.; Mi, A. Q. US 005756799, 1998 [Chem. Abstr. 1998, 129, 54456].

[82] Han, Z. B.; Wang, Z.; Zhang, X. M.; Ding. K. L. Angew. Chem., Int. Ed. 2009, 48, 5345

[83] (a) Wang, X. M.; Han, Z. B.; Wang, Z.; Ding, K. L. Angew. Chem., Int. Ed. 2012, 51, 936.

(b) Wang, X. B.; Guo, P. H.; Wang, X. M.; Wang, Z.; Ding, K. L. Adv. Synth. Catal. 2013, 355, 2900

[84] (a) Wang, X. M.; Meng, F. Y.; Wang, Y.; Han, Z. B.; Chen, Y. J. Angew. Chem., Int. Ed. 2012, 51, 9276.

(b) Cao, Z. Y.; Wang, X.; Tan, C.; Zhao, X. L.; Ding, K. L. J. Am. Chem. Soc. 2013, 135, 8197.

(c) Wang, X. M.; Guo, P. H.; Han, Z. B.; Wang, X. B.; Wang, Z.; Ding, K. L. J. Am. Chem. Soc. 2014, 136, 405.

(d) Liu, J. W.; Han, Z. B.; Wang, X. M.; Ding, K. L. J. Am. Chem. Soc. 2015, 137, 15346

[85] Alain, R.; Francing, A.; Mortreux, A.; Petit, F. Tetrahedron: Asymmetry 1993, 4, 2279.

[86] Alain, R.; Isabelle, S.; Marc, D.; Carpentier, L. F.; Agbossou, F.; Mortreux, A. Tetrahedron: Asymmetry 1996, 7, 379.

[87] Said, N.; Isabell, S.; Mortreux, A.; Agbossou, F.; Mustapha, A. A.; Karim, A. Tetrahedron Lett. 2001, 41, 2867.

[88] Tamao, K.; Yamamoto, H.; Matsumoto, H.; Miyake, N.; Hayashi, T.; Kumda, M. Tetrahedron Lett. 1977, 18, 1389.

[89] Noyori, R.; Ohta, M.; Hsiao, Y.; Kitamura, M.; Ohta, T.; Takaya, H. J. Am. Chem. Soc. 1986, 108, 7117.

[90] Stille, J. K.; Su, H.; Brechot, P.; Parrinello, G. Organometallics 1991, 10, 1123.

[91] Rosini, C.; Franzini, L.; Raffaclli, A. Synthesis 1992, 503.

[92] Zhang, F. Y.; Pai, C. C.; Chan, A. S. J. Am. Chem. Soc. 1998, 120, 5808.

[93] Hu, X. Q.; Chen, H. L.; Zhang, X. M. Angew. Chem., Int. Ed. 1999, 38,3518 .

[94] Ogasawara, M.; Yoshida, K.; Kamei, H.; Kato, K.; Uozumi, Y.; Hayshi, T. Tetrahedron: Asymmetry 1998, 9, 1779.

[95] Imai, Y.; Zhang, W. B.; Kida, T.; Nakatsuji, Y.; Ikeda, I. Tetrahedron Lett. 1998, 39, 4343.

[96] Zhang, Z. G.; Qian, H.; Zhang, X. M. J. Org. Chem. 2000, 65, 6223.

[97] Schmid, R.; Gereghetti, M.; Heiser, B. Helv. Chem. Acta 1988, 71, 897.

[98] Liu, F.; Qian, D. Y.; Li, L.; Zhao, X. L.; Zhang, J. L. Angew. Chem., Int. Ed. 2010, 49, 6669.

[99] Zhang, Z. M.; Chen, P.; Li, W. B.; Niu, Y. F.; Zhao, X. L.; Zhang, J. L. Angew. Chem., Int. Ed. 2014, 53, 4350.

[100] Duclos, M. C.; Singjunla, Y.; Petit, C.; Favre, R. A.; Jeanneau, E.; Popowycz, F.; Lemaire, M. Tetrahedron Lett. 2012, 53, 5984.

[101] Yan, Y. J.; Zhang, X. M. J. Am. Chem. Soc. 2006, 128, 7198.

[102] Desponds, O.; Schlosser, M. J. Organomet. Chem. 1996, 507, 257.

[103] Jendralla, H.; Li, C. H.; Paulaus, E. Tetrahedron: Asymmetry 1994, $5,1297$.

[104] Schimid, R.; Broger, E. A.; Cereghetti, M. Pure Appl. Chem. 1996, 68,131 .
[105] Murata, M.; Morimoto, T.; Achiwa, K. Synlett 1991, 827.

[106] Svensson, G.; Albertsson, J.; Frejd, T. Acta Crystallogr. 1986, 5324.

[107] Schimid, R.; Foricher, J.; Cereghetti, M.; Schonholzer, P. Helv. Chim. Acta 1991, 74, 370.

[108] Yamamoto, W.; Murata, M.; Morimoto, S.; Achiwa, K. Chem. Pharm. Bull. 1991, 39, 1085.

[109] Schmid, R.; Ceregheti, M.; Heiser, B.; Schonholzer, P.; Hansan, H. J. Helv. Chim. Acta 1988, 71, 897.

[110] Sun, X. F.; Zhou, L.; Li, W.; Zhang, X. M. J. Org. Chem. 2008, 73, 1143.

[111] Liu, T. L.; Li, W.; Wang, C. J.; Zhang, X. M. Org. Lett. 2013, 15, 1740 .

[112] (a) Wang, C. J.; Gao, F.; Liang, G. Org. Lett. 2008, 10, 4711. (b) Wang, C. J.; Liang, G.; Xue, Z. Y.; Gao, F. J. Am. Chem. Soc. 2008, 130, 17250 .

[113] Xue, Z. Y.; Liu, T. L.; Lu, Z.; Wang, C. J. Chem. Commun. 2010, 46, 1727.

[114] Li, Q. H.; Tong, M. C.; Li, J.; Wang, C. J. Chem. Commun. 2011 47,11110 .

[115] Li, Q. H.; Liu, T. L.; Zhou, X.; Wang, C. J. Chem. Commun. 2013, 49, 9642.

[116] Liu, H. C.; Tao, H. Y.; Cong, H. J.; Wang. C. J. J. Org. Chem.2016, 81,3751 .

[117] Benincori, T.; Brenna, E.; Sannicolo, F. J. Org. Chem. 1997, 62 , 444.

[118] Benincori, T.; Brenna, E.; Sannicolo, F.; Trimarco, L.; Antognazza, P.; Cesarotti, E.; Demartin F.; Pilati, T. J. Org. Chem. 1996, 61, 6244.

[119] Gelpke, A. E. S.; Kooijman, H.; Spek, A. L.; Hiemstra, H. Chem. Eur. J. 1999, 5, 2472.

[120] Tietze, L. F.; Thede, K.; Sannicolo, F. Chem. Commun. 1999, 1811.

[121] Berens, U.; Brown, J. M.; Long, J.; Selke, R. Tetrahedron: Asymmetry 1996, 7, 285.

[122] McCarthy, M.; Guiry, P. J. Tetrahedron 1999, 55, 3061.

[123] Marquarding, D.; Klusaak, H.; Gokel, G.; Hoffmann, P.; Ugi, I. J. Am. Chem. Soc. 1970, 92, 5389.

[124] Richards, C. J.; Locke, A. J. Tetrahedron: Asymmetry 1998, 9, 2377.

[125] You, S. L.; Hou, X. L.; Dai, L. X.; Yu, Y. H.; Xia, W. J. Org. Chem. 2002, 67, 4684.

[126] Mathey, F.; Mercier, F.; Robin, F.; Ricard, L. J. Organoment. Chem. 1998, 557, 117.

[127] Hayashi, T.; Yamamoto, A.; Hojo, M. J. Am. Chem. Soc. 1982, 104, 180.

[128] (a) Ogasawara, M.; Yoshida, K.; Hayashi, T. Organometallics 2001, 20,1014 .

(b) Ogasawara, M.; Yoshida, K.; Hayashi, T. Organometallics 2001, $20,3913$.

[129] (a) Wang, M. C.; Xu, C. L.; Zou, Y. X.; Liu, H. M.; Wang, D. K. Tetrahedron Lett. 2005, 46, 5413.

(b) Wang, M. C.; Liu, L. T.; Hua, Y. Z.; Zhang, J. S.; Shi, Y. Y.; Wang, D. K. Tetrahedron: Asymmetry 2005, 16, 2531.

[130] Li, G. W.; Tang, B.; Wang, M. C.; Kong, P. P.; Zhao, W. X. Chin. J. Synth. Chem. 2012, 20, 462 (in Chinese). (李高伟, 唐贝，王敏灿，孔培培，赵文献，合成化学，2012，20, 462.)

[131] Zhang, A. A.; Zhao, R. J.; Chen, R. X.; Kong, W. G.; Zhang, P. F.; Zhao, W. X. Shangqiu Normal College J. 2013, 29, 50 (in Chinese). (张安安, 赵瑞娟, 陈荣祥, 孔伟光, 张朋飞, 赵文献, 商丘师范 学院学报, 2013, 29, 50.)

[132] Pfaltz, A. Acta Chem. Scand B 1996, 50, 189

[133] Reetz, M. T.; Gosberg, A.; Goddard, R.; Kyung, S. H. Chem. Commun. 1998, 2077. 
[134] (a) Chi, Y. X.; Zhou, Y. G.; Zhang, X. M. J. Org. Chem. 2003, 68, 4120 .

(b) Hou, G. H.; Li, W.; Ma, M. F.; Zhang, X. M. J. Am. Chem. Soc. 2010, 132, 12844.

[135] Zhao, Q. Y.; Li, S. K.; Huang, K. X.; Zhang, X. M. Org. Lett. 2013 , 15,4014 .

[136] Li, P.; Zhou, M.; Zhao, Q. Y.; Wu, W. L.; Zhang, X. M. Org. Lett. 2016, $18,40$.

[137] Reetz, M. T.; Gosberg, A.; Goddard, R.; Kyung, S. H. Chem. Commun. 1998, 2077.

[138] Kless, A.; Lefber, C.; Spannenberg, A.; Kempe, R.; Baumann, W.; Hola, J.; Armin, B. Tetrahedron 1996, 52, 14599.

[139] Mayer, H. A.; Otto, H.; Kuhbauch, H. J. Organomet. Chem. 1994, $472,347$.

[140] Delapierre, G.; Brurel, J. M.; Constantieux, T.; Buono, G. Tetrahedron: Asymmetry 2001, 12, 1345.

[141] Reeta, M. T.; Beuttenmuller, E. W.; Goddard, R. Tetrahedron Lett. 1997, 38, 3211.

[142] Zhu, G. X.; Cao, P.; Jiang, Q. Z.; Zhang, X. M. J. Am. Chem. Soc. 1997, 119, 1799

[143] Alexakis, A.; Burton, J.; Vastra, J.; Mangeney, P. Tetrahedron: Asymmetry 1997, 8, 3987.

[144] Brunel, J. M.; Constantieux, T.; Legrand, O.; Buono, G. Tetrahedron Lett. 1998, 39, 2961.

[145] Sugihara, H.; Daikai, K.; Jin, X. L.; Furuno, H.; Inanaga, J. Tetrahedron Lett. 2002, 43, 2735.

[146] (a) Fan, Q. H.; Chen, Y. M.; Chen, X. M. Chem. Commun. 2000, 789.

(b) Yi, B.; Chem, X. M.; Deng, G. J. Org. Lett. 2004, 6, 1361.

(c) Ma, B. D.; Deng, G. J.; Fan, Q. H. Acta Chim. Sinica 2013, 71, 528 (in Chinese).

(马保德，邓国军，范青华，化学学报，2013，71，528.)

[147] Cheng, K. J. M.S. Thesis, Lanzhou Institute of Chemical Physics, Chinese Academy of Sciences, Lanzhou, 1994 (in Chinese). (成克军, 硕士论文, 中国科学院兰州化学物理研究所, 兰州, 1994.)

[148] Hayashi, T.; Kawamara, N.; Ito, Y. J. Am. Chem. Soc. 1987, 109, 7876.

[149] Sayo, N.; Taketomi, T.; Kumobayashi, H. EP 271311, 1988 [Chem. Abstr. 1989, 110, 39189].
[150] Zheng. X. L.; Wang, Y. H.; Zuo, H. P.; He, H. P. J. Mol. Catal. 1996, 10, 70 (in Chinese).

(郑晓来，王艳华，左焕培，何华平，分子催化, 1996, 10, 70.)

[151] Parrinello, G.; Stille, J. K. J. Am. Chem. Soc. 1987, 109, 7122.

[152] Ishizaki, T. EP 5444551993 [Chem. Abstr. 1993, 119, 181016.]

[153] Dumont, W.; Poalin, J. C.; Dang, T. P.; Kagan, H. B. J. Am. Chem. Soc. 1973, 95, 8295.

[154] Takaishi, N.; Jmai, H.; Bertelo, C. A.; Stille, J. K. J. Am. Chem. Soc. 1978, 100, 264.

[155] Poulin, J. C.; Dumont, W.; Dang, T. P.; Kagan, H. B. Compt. Rend. Acad. Sci. Paris 1973, 227C, 41

[156] Stille, J. K. React. Polym. 1989, 10, 165.

[157] Yi, B.; Wu, G. F.; Zhou, W. Chin. J. Org. Chem. 2013, 33, 2143 (in Chinese).

(易兵, 武高峰，周威，有机化学, 2013, 33, 2143.)

[158] Wan, K. T.; Davis, M. E. Nature 1994, 370, 449.

[159] Cheng, Z. B.; Yu, S. L.; Li, Y. Y. Chem. Res. Chin. Univ. 2011, 27, 170.

[160] Yu, S. L.; Li, Y. Y.; Dong, Z. R.; Zhang, J. N.; Li, Q.; Gao, J. X. Chin. Chem. Lett. 2011, 22, 1269

[161] Zhang, J. N.; Yang, X. R.; Zhou, H.; Li, Y. Y.; Dong, Z. R.; Gao, J. X. Green Chem. 2012, 14, 1289.

[162] Xu, Y. Q.; Yu, S. L.; Li, Y. Y.; Dong, Z. R.; Gao, J. X. Chin. Chem. Lett. 2013, 24, 527.

[163] Zeng, L.; Wu, F.; Li, Y. Y. J. Org. Chem. 2014, 762, 34.

[164] Trost, B. M.; Brendan, M. Org. Lett. 2008, 10, 1369.

[165] Xie, J. H.; Zhou, Q. L. Acta Chim. Sinica 2014, 72, 778 (in Chinese). (谢建华, 周其林, 化学学报, 2014, 72, 778.)

[166] (a) Hu, A. G.; Fu, Y.; Xie, J. H.; Zhou, Q. L. Angew. Chem., Int. Ed. 2002, 41, 2348.

(b) Fu, Y.; Xie, J. H.; Hu, A. G.; Zhou, H.; Wang, L. X.; Zhou, Q. L. Chem. Commun. 2002, 480.

[167] (a) Xie, J. H.; Wang, L. X.; Fu, Y.; Zhu, S. F.; Fan, B. M.; Duan, H. F.; Zhou, Q. L. J. Am. Chem. Soc. 2003, 125, 4404.

(b) Zhu, G. X.; Cao, P.; Jiang, Q. Z.; Zhang, X. M. J. Am. Chem. Soc. 1997, 119, 1799

[168] Han, Z. B.; Wang, Z.; Zhang, X. M.; Ding, K. Angew. Chem., Int. Ed. 2009, $48,5345$. 